\title{
Hopf Bifurcation Characteristics of Dual-Front Axle Self-Excited Shimmy System for Heavy Truck considering Dry Friction
}

\author{
Daogao Wei, ${ }^{1}$ Ke Xu, ${ }^{1}$ Yibin Jiang, ${ }^{1}$ Changhe Chen, ${ }^{1}$ Wenjing Zhao, ${ }^{1}$ and Fugeng Zhou ${ }^{2}$ \\ ${ }^{1}$ School of Mechanical and Automotive Engineering, Hefei University of Technology, Hefei 230009, China \\ ${ }^{2}$ China Anhui Jianghuai Automobile Co., Ltd., Hefei 230022, China \\ Correspondence should be addressed to Daogao Wei; weidaogao@hfut.edu.cn
}

Received 19 April 2015; Accepted 13 August 2015

Academic Editor: Marcello Vanali

Copyright (c) 2015 Daogao Wei et al. This is an open access article distributed under the Creative Commons Attribution License, which permits unrestricted use, distribution, and reproduction in any medium, provided the original work is properly cited.

\begin{abstract}
Multiaxle steering is widely used in commercial vehicles. However, the mechanism of the self-excited shimmy produced by the multiaxle steering system is not clear until now. This study takes a dual-front axle heavy truck as sample vehicle and considers the influences of mid-shift transmission and dry friction to develop a 9 DOF dynamics model based on Lagrange's equation. Based on the Hopf bifurcation theorem and center manifold theory, the study shows that dual-front axle shimmy is a self-excited vibration produced from Hopf bifurcation. The numerical method is adopted to determine how the size of dry friction torque influences the Hopf bifurcation characteristics of the system and to analyze the speed range of limit cycles and numerical characteristics of the shimmy system. The consistency of results of the qualitative and numerical methods shows that qualitative methods can predict the bifurcation characteristics of shimmy systems. The influences of the main system parameters on the shimmy system are also discussed. Improving the steering transition rod stiffness and dry friction torque and selecting a smaller pneumatic trail and caster angle can reduce the self-excited shimmy, reduce tire wear, and improve the driving stability of vehicles.
\end{abstract}

\section{Introduction}

In recent years, with the rapid development of the transportation industry, high-speed and multiaxle heavy trucks with dual-front axles have become widely used for their load capacity, high performance-price ratio, adaptability, and high horsepower. The dual-front axle steering system is a relatively advanced steering system given its low cost, simple handling, steering safety and stability under heavy load, and the less harm it causes on the road surface during driving. However, the shimmy problem in its dual-front axle steering system leads to abnormal tire wear (especially the tire on the second axle), off-tracking, and shaking of the steering wheel [1-3].

Research on the shimmy of single-axle vehicles can be traced back to 80 years ago and can be classified into forced shimmy and self-excited shimmy, which is a Hopf bifurcation phenomenon. Extensive research has been conducted on this field and can be used to solve the shimmy problems in engineering [4-7]. Given the growth in the demand for multiaxle heavy trucks, current research on the dual-axle steering system shimmy has demonstrated its significance.
Considering its sophisticated mechanism, the shimmy of a dual-axle steering system differs from that of a singleaxle steering system. Therefore, many scholars have studied the problem extensively. Watanabe et al. [1] studied the effect of the number and position of driving wheels on the steering performance of dual-axle steering vehicles. Gu et al. [8] demonstrated the content and method in the design of heavy trucks with a multiaxle steering system, analyzed the main problems in this research field in China, and proposed a design method for a steering system based on integrated and optimized matching platform for a chassis system. Hou et al. [9] established the kinematics model and a mathematical optimization model for the multiaxle steering system of $10 \times 8$ heavy-duty vehicle and designed a new weight function considering the probability of steering angle. The parameters of multiaxle steering system were optimized. The result showed that the result with weight function had better effect than other conditions. Wang et al. [10] applied a robust design theory with design parameters and noise factors following a normal distribution in a dual-front axle steering system. He combined reliability optimization 
with robust design and built a mathematical model for the robust reliability optimization of dual-front axles with clearances. Xu et al. [11] developed a steering wheel shimmy model for a four-axis steering crane without considering the nonlinear factors and their impact on the four-axis steering crane shimmy. Nisonger and Wormley [12] compared the transverse dynamic characteristics of single- and dual-front axle steering systems, using a nonlinear model with three degrees of freedom. Wu and Lin [13] found that the doublefront axle can improve a car's yaw stability. Williams [14] extended the dual-axle model for vehicles to a multiaxle model and analyzed its steering ability and handling stability. By analyzing a linear dual-front axle yaw dynamics model, Demić [15] analyzed the influence of structural parameters on the front wheel shimmy of a heavy vehicle and found that the vibration of suspension can cause front wheel shimmy. Cole and Cebon [16] developed a vibration model for the pneumatic suspension of a heavy truck to reduce the vibration in and damage to the road surface caused by trucks through suspension parameter optimization. Chen [17] concluded that two reasons-internal and externalaccount for abnormal tire wear, proposed improvement measures based on his practical experience, and pointed out directions for future research. Liao [18] and Li et al. [19] explored the application of multirigid body theory in the dynamic simulation of a dual-front axle steering system and proposed the condition under which tires bear heavy load in a dual-front axle vehicle. They also built a spatial model for the dual-front axle based on ADAMS and conducted a simulation analysis. Using TruckSim, a dynamics analysis software for vehicles, $\mathrm{Li}$ [20] studied how to determine and describe the seven characteristic parameters of a heavy truck steering system, namely, axle and suspension, transmission, tire, body, brake system, and aerodynamics, and laid down a solid foundation for the study of multiaxle steering technology and the improvement of its handling stability.

Most of the aforementioned studies focus on the constructional parameter analysis and simulation of dual-front axle steering system; however, there is little research on steering system shimmy, especially there is little research on the mechanism of Hopf bifurcation produce self-shimmy. But it is not yet clear about Hopf bifurcation characteristic of dual-front axle self-shimmy for heavy truck. Therefore, the research in this area is very important. In this study, we took a JAC heavy truck as an example and established nine degrees of freedom equations for the dual-front axle steering system based on Lagrange's equation. We determined the existence and the value of Hopf bifurcation with the use of the Hurwitz criterion [21] and Hopf bifurcation theorem. To obtain the manifold for the system in two-dimensional space, we reduced the dimension of the differential equations of state using center manifold theory and found the Hopf paradigm in the polar coordinates with the use of normative theory so that the Hopf bifurcation characteristics of the shimmy system of the dual-front axles can be fully analyzed. Based on the above analysis, we then propose a method of controlling such shimmy. As such, this study establishes the theoretical foundation for research on the mechanism of the dual-front axle self-excited shimmy and provides

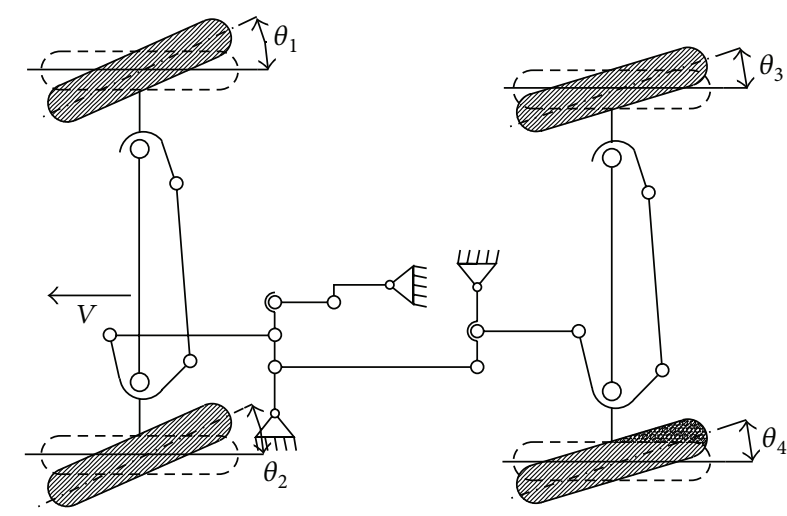

FIGURE 1: Schematic of dual-front axle steering system.

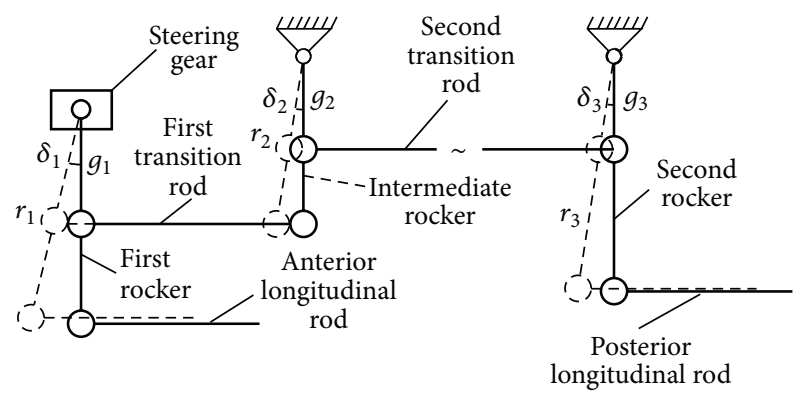

FIGURE 2: Schematic of intermediate steering transmission mechanism.

a reference on the design improvement of steering systems. The contribution of this study is the finding that the selfexcited shimmy phenomenon of the dual-front axle of a heavy truck is generated by Hopf bifurcation.

\section{Dynamics Model for a Dual-Front Axle Shimmy System}

2.1. Mechanical Model. The schematic of the structure of the widely used dual-front axle steering system is shown in Figure 1; the diagram of the steering intermediate transmission is shown in Figure 2. To facilitate the development of the mathematical model of the dual-front axle self-excited shimmy system, the mechanics model of the dual-front axle self-excited shimmy system is developed based on Figure 2, with the dual-front axle steering system of a JAC heavy truck as prototype, the schematics of which are shown in Figure 3. Figure 3 illustrates the double-front axle steering principle: the steering force exerted by the driver is passed through the steering operating mechanism, which is composed of the steering shaft, transmission shaft, and steering joints, towards the steering gear (2). The torque is then transmitted to the first rocker (3) after its torque increases and its speed decreases. The first rocker (3) then drives the steering knuckle arm (5) of the first steering bridge through the anterior longitudinal rod (4) to turn the left wheel to rotate around the kingpin, which is installed in the first steering bridge. At the same time, the right wheel driven by the torque, which passes 


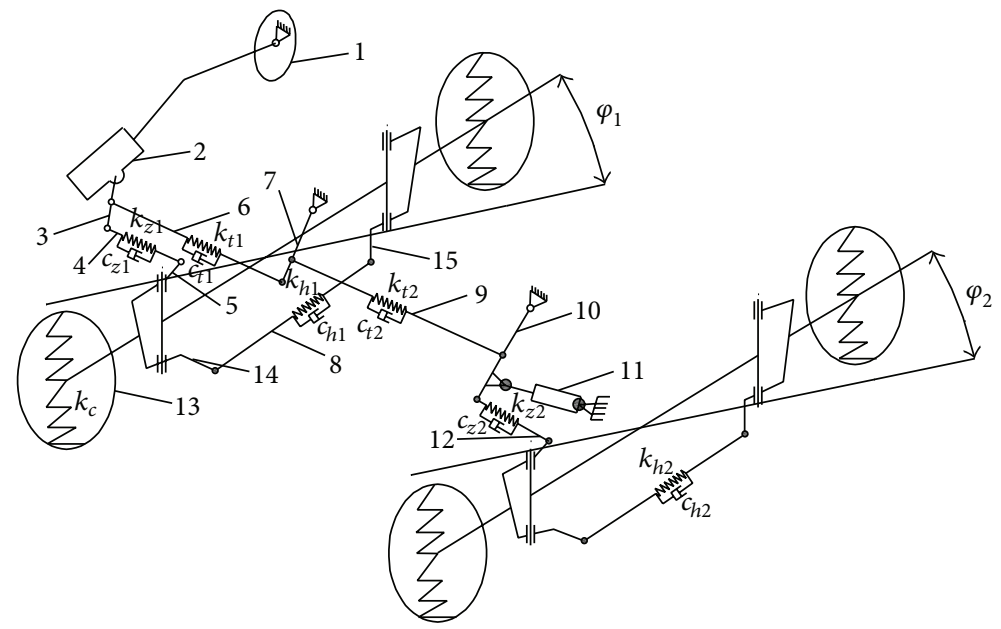
(1) Steering wheel
(2) Steering gear
(9) Second transition rod
(3) First rocker
(4) Anterior longitudinal rod
(10) Second rocker
(5) Steering knuckle arm
(11) Steering power cylinder
(12) Posterior longitudinal rod
(6) First transition rod
(13) Tire
(7) Intermediate rocker
(14) Left trapezoid arm
(8) Tie rod
(15) Right trapezoid arm

FIGURE 3: Dual-front axle shimmy system mechanics model.

through the left trapezoid arm (14) to the tie rod (8) to the right trapezoid arm (15), rotates. The second bridge steering is consistent with the first bridge steering, and the steering force is passed through the first transition rod (6) to the intermediate rocker (7) to the second transition rod (9) to the second rocker (10) to the posterior longitudinal rod (12) and to the second bridge.

In the mechanical model, the elasticity of the rod is considered; it is equivalent to a spring-damper unit. In line with the law of the right-hand coordinate system, the center of the mass of the vehicle is deemed as the coordinate origin; the vehicle forward direction is for $x$-axis, the vehicle left direction is for $y$-axis, and perpendicular to the ground up direction is for $z$-axis. Figure 3 shows that the system has nearly 20 rotating hinges. To facilitate the dynamic analysis and mathematical modeling and to highlight the effect of multisport hinges, dry friction, and other parameters of the self-excited shimmy system, as shown in Figure 3, the following assumptions are made to establish a dynamics model for the dual-front axle steering shimmy system: (1) The steering wheel is immobile. (2) The impact of the force of air is ignored. (3) The various parts associated with the vibration and their couplings are simplified according to the moments of inertia, springs, and dampers. (4) The angle between the steering trapezoid plane and $X Y$ plane and the angle between the steering linkage and the $X Z$ plane are ignored. (5) The direction and speed of the vehicle is constant. (6) Longitudinal and lateral slips do not occur in the vehicle. (7) The dry friction in the kinematic pairs is equivalent to the kingpin of the first or second bridge.

2.2. The Equation for the Motion of the Shimmy System. According to the dynamics model in Figure 3, we established the equations for the motion of the dual-front axle steering shimmy system of heavy vehicles. The shimmy system has nine degrees of freedom: $\theta_{1}$ is the swing angle at which the left wheel of the first bridge rotates around the kingpin. $\theta_{2}$ is the swing angle at which the right wheel of the first bridge rotates around the kingpin. $\varphi_{1}$ is the lateral swing angle of the first bridge. $\theta_{3}$ is the swing angle at which the left wheel of the second bridge rotates around the kingpin. $\theta_{4}$ is the swing angle at which the right wheel of the second bridge rotates around the kingpin. $\varphi_{2}$ is the lateral swing angle of the second bridge. $\delta_{1}$ is the swing angle of the first rocking arm. $\delta_{2}$ is the swing angle of the intermediate steering arm. $\delta_{3}$ is the swing angle of the second rocking arm.

In this study, the mathematical model of the shimmy system of the sample vehicle is established using Lagrange's equations, which can be expressed as

$$
\begin{array}{r}
\frac{d}{d t}\left(\frac{\partial T}{\partial \dot{q}_{k}}\right)-\frac{\partial T}{\partial q_{k}}+\frac{\partial U}{\partial q_{k}}+\frac{\partial D}{\partial \dot{q}_{k}}=Q_{k} \\
(k=1,2,3, \ldots, 9),
\end{array}
$$

where $q_{k}$ represents nine degrees of freedom of the system, $T$ represents the system's kinetic energy, $U$ represents the system's potential energy, $D$ represents the system's dissipated energy, and $Q_{k}$ represents the nine generalized forces to which the system is subjected.

According to Figure 3, kinetic energy, potential energy, dissipated energy, and the nine generalized forces of the dualfront axle steering shimmy system are obtained as follows. 
The kinetic energy of the shimmy system is

$$
\begin{aligned}
T= & \frac{1}{2} I_{1}\left(\dot{\theta}_{1}^{2}+\dot{\theta}_{2}^{2}\right)+\frac{1}{2} J_{1} \dot{\varphi}_{1}^{2}+\frac{1}{2} I_{2}\left(\dot{\theta}_{3}^{2}+\dot{\theta}_{4}^{2}\right)+\frac{1}{2} J_{2} \dot{\varphi}_{2}{ }^{2} \\
& +\frac{1}{2} I_{c 1} \dot{\delta}_{1}^{2}+\frac{1}{2} I_{c 2} \dot{\delta}_{2}^{2}+\frac{1}{2} I_{c 3} \dot{\delta}_{3}^{2},
\end{aligned}
$$

where $I_{i}(i=1,2)$ is the moment of inertia of the wheel around the kingpin in $i$ th bridge, $I_{c i}(i=1,3)$ is the moment of inertia of $i$ th rocker, $I_{c 2}$ is the moment of inertia of the intermediate rocker, and $J_{i}(i=1,2)$ is the moment of inertia of $i$ th bridge around its side off-axis.

The potential energy of the shimmy system is

$$
\begin{aligned}
U= & \frac{1}{2} k_{h 1}\left(\theta_{1}-\theta_{2}\right)^{2}+\frac{1}{2} k_{n} \varphi_{1}^{2}+\frac{1}{2} k_{h 2}\left(\theta_{3}-\theta_{4}\right)^{2} \\
& +\frac{1}{2} k_{n} \varphi_{2}^{2}+\frac{1}{2} k_{t} \delta_{1}^{2}+\frac{1}{2} k_{t 1}\left(g_{1} \delta_{1}-r_{2} \delta_{2}\right)^{2} \\
& +\frac{1}{2} k_{t 2}\left(g_{2} \delta_{2}-g_{3} \delta_{3}\right)^{2}+\frac{1}{2} k_{z 1}\left(r_{1} \delta_{1}+a_{1} \theta_{2}\right)^{2} \\
& +\frac{1}{2} k_{z 2}\left(r_{3} \delta_{3}+a_{2} \theta_{4}\right)^{2},
\end{aligned}
$$

where $k_{h i}(i=1,2)$ is the stiffness of the tie rod converted into the stiffness around the kingpin; $k_{n}$ is the equivalent angle stiffness of the suspension converted into the side swing center; $k_{t}$ is the inverse stiffness of the first rocking arm to the steering wheel; $k_{t i}(i=1,2)$ is the stiffness of $i$ th transition rod; $k_{z i}(i=1,2)$ is the stiffness of the front and rear longitudinal rods; $r_{1}$ is the effective length of the first arm; $r_{2}$ is the effective length of the middle arm; $r_{3}$ is the effective length of the second arm; $g_{1}, g_{2}$, and $g_{3}$ are the distances between the pivot point of the rocker and the transition lever and the fixed end of the rocker arm; and $a_{i}(i=1,2)$ is the distance between the hinge point of $i$ th bridge knuckle arm and vertical rod and kingpin.

The dissipated energy of the shimmy system is

$$
\begin{aligned}
D= & \frac{1}{2} c_{l 1}\left(\dot{\theta}_{1}+\dot{\theta}_{2}\right)+\frac{1}{2} c_{h 1}\left(\dot{\theta}_{1}-\dot{\theta}_{2}\right)^{2}+\frac{1}{2} c_{n} \dot{\varphi}_{1}^{2} \\
& +\frac{1}{2} c_{h 2}\left(\dot{\theta}_{3}-\dot{\theta}_{4}\right)^{2}+\frac{1}{2} c_{l 2}\left(\dot{\theta}_{3}+\dot{\theta}_{4}\right)+\frac{1}{2} c_{n} \dot{\varphi}_{2}^{2} \\
& +\frac{1}{2} c_{t} \dot{\delta}_{1}^{2}+\frac{1}{2} c_{t 1}\left(g_{1} \dot{\delta}_{1}-r_{2} \dot{\delta}_{2}\right)^{2} \\
& +\frac{1}{2} c_{t 2}\left(g_{2} \dot{\delta}_{2}-g_{3} \dot{\delta}_{3}\right)^{2}+\frac{1}{2} c_{z 1}\left(r_{1} \dot{\delta}_{1}+a_{1} \dot{\theta}_{2}\right)^{2} \\
& +\frac{1}{2} c_{z 2}\left(r_{3} \dot{\delta}_{3}+a_{2} \dot{\theta}_{4}\right)^{2}+M_{22} \dot{\theta}_{4}+M_{21} \dot{\theta}_{3}+M_{12} \dot{\theta}_{2} \\
& +M_{11} \dot{\theta}_{1},
\end{aligned}
$$

where $c_{l i}(i=1,2)$ is the equivalent damping of $i$ th wheel around the kingpin, $c_{h i}(i=1,2)$ is the damping of the tie rod converted into the damping around the kingpin, $c_{n}$ is the equivalent damping of the suspension converted into the side swing center, $c_{t}$ is the inverse damping of the first rocking arm to the steering wheel, $c_{t i}(i=1,2)$ is the damping of $i$ th transition rod, $c_{z i}(i=1,2)$ is the damping of the front and rear longitudinal rods, $M_{11}$ is the equivalent friction torque of the first bridge at the right wheel kingpin, $M_{12}$ is the equivalent friction torque of the first bridge at the left wheel kingpin, $M_{21}$ is the equivalent friction torque of the second bridge at the right wheel kingpin, and $M_{22}$ is the equivalent friction torque of the second bridge at the left wheel kingpin.

The nine generalized forces of the shimmy system are as follows; for details about the procedure for calculation of the generalized forces see appendix:

$$
\begin{aligned}
Q_{1}= & -i_{k 1} \frac{v}{R} \dot{\varphi}_{1}+F_{1}(R \gamma+e) \\
& -\left(\gamma \rho h_{1} R+\frac{k_{c}}{2} B_{1} l_{1}(-f+\gamma)\right) \varphi_{1}, \\
Q_{2}= & -i_{k 1} \frac{v}{R} \dot{\varphi}_{1}+F_{2}(R \gamma+e) \\
& -\left(\gamma \rho h_{1} R+\frac{k_{c}}{2} B_{1} l_{1}(-f+\gamma)\right) \varphi_{1}, \\
Q_{3}= & -2 \rho R h_{1} \varphi_{1}+i_{k 1} \frac{v}{R}\left(\dot{\theta}_{1}+\dot{\theta}_{2}\right)-\left(F_{1}+F_{2}\right) R, \\
Q_{4}= & -i_{k 2} \frac{v}{R} \dot{\varphi}_{2}+F_{3}(R \gamma+e) \\
& -\left(\gamma \rho h_{2} R+\frac{k_{c}}{2} B_{2} l_{2}(-f+\gamma)\right) \varphi_{2}, \\
Q_{5}= & -i_{k 2} \frac{v}{R} \dot{\varphi}_{2}+F_{4}(R \gamma+e) \\
Q_{8}= & 0, \quad\left(\gamma \rho h_{2} R+\frac{k_{c}}{2} B_{2} l_{2}(-f+\gamma)\right) \varphi_{2}, \\
Q_{9}= & 0, \\
Q_{6}= & -2 \rho R h_{2} \varphi_{2}+i_{k 2} \frac{v}{R}\left(\dot{\theta}_{3}+\dot{\theta}_{4}\right)-\left(F_{3}+F_{4}\right) R, \\
& 0,
\end{aligned}
$$

where $i_{k i}(i=1,2)$ is the moment of inertia of the wheel around its own axis of rotation in $i$ th bridge, $v$ is the vehicle speed, $R$ is the rolling radius of the tire, $F_{1}$ is the first bridge right wheel subjected to lateral force, $F_{2}$ is the first bridge left wheel subjected to lateral force, $F_{3}$ is the second bridge right wheel subjected to lateral force, $F_{4}$ is the second bridge left wheel subjected to lateral force, $\gamma$ is the kingpin caster angle of the wheel, $e$ is the pneumatic trail, $\rho$ is the tire lateral stiffness, $h_{i}$ is the height of $i$ th suspension roll center $(i=1,2), k_{c}$ is the tire vertical stiffness, $B_{i}(i=1,2)$ is the tread in $i$ th bridge, $l_{i}(i=1,2)$ is the distance between the point of the kingpin extension line with the ground intersection and plane of symmetry of the wheel, $f$ is the friction coefficient between the tire and the ground, and $\sigma$ is the tire relaxation length.

According to (2) to (5), the kinetic equations for the system are derived from Lagrange's equations, as given by (1). 
Equations for the motion of the right wheel of the first bridge around the kingpin are as follows:

$$
\begin{aligned}
I_{1} \ddot{\theta}_{1}+ & c_{l 1} \dot{\theta}_{1}+k_{h 1}\left(\theta_{1}-\theta_{2}\right)+c_{h 1}\left(\dot{\theta}_{1}-\dot{\theta}_{2}\right)+\frac{i_{k 1} v}{R} \dot{\varphi}_{1} \\
& +\left(\rho R h_{1} \gamma+\frac{1}{2} k_{c} l_{1} B_{1}(\gamma-f)\right) \varphi_{1}-F_{1}(R \gamma+e) \\
& +M_{11}=0 .
\end{aligned}
$$

Equations for the motion of the left wheel of the first bridge around the kingpin are as follows:

$$
\begin{aligned}
I_{1} \ddot{\theta}_{2} & +c_{l 1} \dot{\theta}_{2}+k_{h 1}\left(\theta_{2}-\theta_{1}\right)+c_{h 1}\left(\dot{\theta}_{2}-\dot{\theta}_{1}\right) \\
& +k_{z 1} a_{1}\left(r_{1} \delta_{1}+a_{1} \theta_{2}\right)+c_{z 1} a_{1}\left(r_{1} \dot{\delta}_{1}+a_{1} \dot{\theta}_{2}\right) \\
& +\frac{i_{k 1} v}{R} \dot{\varphi}_{1}+\left(\rho R h_{1} \gamma+\frac{1}{2} k_{c} l_{1} B_{1}(\gamma-f)\right) \varphi_{1} \\
& -F_{2}(R \gamma+e)+M_{12}=0 .
\end{aligned}
$$

Lateral swing equations for the motion of the first bridge are as follows:

$$
\begin{array}{r}
J_{1} \ddot{\varphi}_{1}+c_{n 1} \dot{\varphi}_{1}+\left(\frac{1}{2} k_{c} B_{1}{ }^{2}+k_{n}+2 \rho R h_{1}\right) \varphi_{1} \\
-\frac{i_{k 1} v}{R}\left(\dot{\theta}_{1}+\dot{\theta}_{2}\right)+\left(F_{1}+F_{2}\right) h_{1}=0 .
\end{array}
$$

Equations for the motion of the right wheel of the second bridge around the kingpin are as follows:

$$
\begin{aligned}
I_{2} \ddot{\theta}_{3}+ & c_{l 2} \dot{\theta}_{3}+k_{h 2}\left(\theta_{3}-\theta_{4}\right)+c_{h 2}\left(\dot{\theta}_{3}-\dot{\theta}_{4}\right)+\frac{i_{k 2} v}{R} \dot{\varphi}_{2} \\
& +\left(\rho R h_{2} \gamma+\frac{1}{2} k_{c} l_{2} B_{2}(\gamma-f)\right) \varphi_{2}-F_{3}(R \gamma+e) \\
& +M_{21}=0 .
\end{aligned}
$$

Equations for the motion of the left wheel of the second bridge around the kingpin are as follows:

$$
\begin{aligned}
I_{2} \ddot{\theta}_{4} & +c_{l 2} \dot{\theta}_{2}+k_{h 2}\left(\theta_{4}-\theta_{3}\right)+c_{h 2}\left(\dot{\theta}_{4}-\dot{\theta}_{3}\right) \\
& +k_{z 2} a_{2}\left(r_{3} \delta_{3}+a_{2} \theta_{4}\right)+c_{z 2} a_{2}\left(r_{3} \dot{\delta}_{3}+a_{2} \dot{\theta}_{4}\right) \\
& +\frac{i_{k 2} v}{R} \dot{\varphi}_{2}+\left(\rho R h_{2} \gamma+\frac{1}{2} k_{c} l_{2} B_{2}(\gamma-f)\right) \varphi_{2} \\
& -F_{4}(R \gamma+e)+M_{22}=0 .
\end{aligned}
$$

Lateral swing equations for the motion of the second bridge are as follows:

$$
\begin{aligned}
J_{2} \ddot{\varphi}_{2} & +c_{n 2} \dot{\varphi}_{2}+\left(\frac{1}{2} k_{c} B_{2}{ }^{2}+k_{n}+2 \rho R h_{2}\right) \varphi_{2} \\
- & \frac{i_{k 2} v}{R}\left(\dot{\theta}_{3}+\dot{\theta}_{4}\right)+\left(F_{3}+F_{4}\right) h_{2}=0 .
\end{aligned}
$$

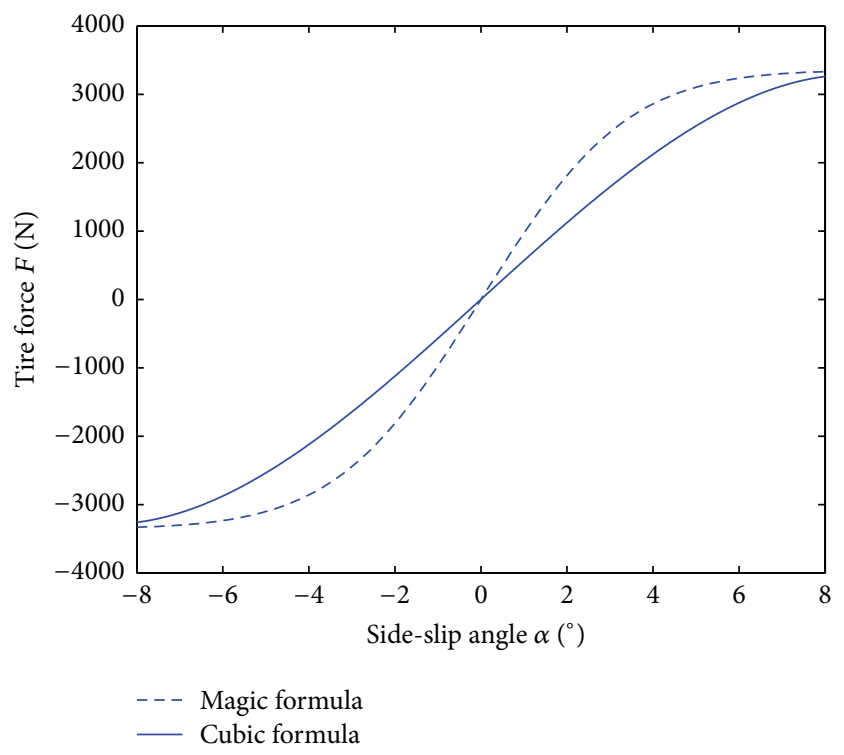

FIgURE 4: Curve of relationship between $F_{i}$ and $\alpha_{i}$.

Swing equations for the motion of the first rocking arm are as follows:

$$
\begin{aligned}
I_{c 1} \ddot{\delta}_{1} & +c_{t} \dot{\delta}_{1}+k_{t} \delta_{1}+c_{t 1} g_{1}\left(g_{1} \dot{\delta}_{1}-r_{2} \dot{\delta}_{2}\right) \\
& +k_{t 1} g_{1}\left(g_{1} \delta_{1}-r_{2} \delta_{2}\right)+c_{z 1} r_{1}\left(r_{1} \dot{\delta}_{1}+a_{1} \dot{\theta}_{2}\right) \\
& +k_{z 1} r_{1}\left(r_{1} \delta_{1}+a_{1} \theta_{2}\right)=0 .
\end{aligned}
$$

Swing equations for the motion of the intermediate steering arm are as follows:

$$
\begin{aligned}
I_{c 2} \ddot{\delta}_{2} & +c_{t 1} r_{2}\left(r_{2} \dot{\delta}_{2}-g_{1} \dot{\delta}_{1}\right)+k_{t 1} r_{2}\left(r_{2} \delta_{2}-g_{1} \delta_{1}\right) \\
& +c_{t 2} g_{2}\left(g_{2} \dot{\delta}_{2}-g_{3} \dot{\delta}_{3}\right)+k_{t 2} g_{2}\left(g_{2} \delta_{2}-g_{3} \delta_{3}\right) \\
= & 0 .
\end{aligned}
$$

Swing equations for the motion of the second rocking arm are as follows:

$$
\begin{aligned}
I_{c 3} \ddot{\delta}_{3} & +c_{t 2} g_{3}\left(g_{3} \dot{\delta}_{3}-g_{2} \dot{\delta}_{2}\right)+k_{t 2} g_{3}\left(g_{3} \delta_{3}-g_{2} \delta_{2}\right) \\
& +c_{z 2} r_{3}\left(r_{3} \dot{\delta}_{3}+a_{2} \dot{\theta}_{4}\right)+k_{z 2} r_{3}\left(r_{3} \delta_{3}+a_{2} \theta_{4}\right)=0 .
\end{aligned}
$$

2.3. Tire Model Selection. Several nonlinear tire models are commonly used in the simulation of vehicle dynamics, such as Pacejka's magic formula, cube model, Guo Konghui's semiempirical tire theoretical model, and Gim's tire models [2225]. Equations (15) and (16) are the mathematical expressions of the cube and magic formula models, respectively. The cornering force curves of these two tire models are shown in Figure 4, which shows that two cornering force curves have the same trend and that the cube model is similar to the magic formula. Moreover, the cube model is simple; it does not require a considerable amount of experimental data, can precisely reveal the performance characteristic trends of 
TABLE 1: Parameters of tire model.

\begin{tabular}{cccccccccc}
\hline$C_{1 f}$ & $C_{3 f}$ & $a_{1}$ & $a_{2}$ & $a_{3}$ & $a_{4}$ & $a_{5}$ & $a_{6}$ & $a_{7}$ & $a_{8}$ \\
\hline 32740 & 481770 & -22.1 & 1011 & 1078 & 1.82 & 0.208 & 0 & -0.354 & 0.707 \\
\hline
\end{tabular}

the tire force, and does not contain trigonometric functions, thereby facilitating qualitative analysis. As such, we choose the cube model as the tire model in this study. The cube model is the third-order truncation of the magic formula [23]. The coefficients $\left(B_{i}, D_{i}\right.$, and $\left.E_{i}\right)$ of magic formula take into account the impact of the vertical load $F_{Z i}$. So the cube model cube considers the vertical force $F_{Z i}$ :

$$
\begin{aligned}
F_{i} & =-\left(C_{1 f} \alpha_{i}-C_{3 f} \alpha_{i}^{3}\right), \quad(i=1,2,3,4), \\
F_{i} & =D_{i} \sin \left(C_{i}\right. \\
& \left.\times \arctan \left(B_{i} a_{i}-E_{i}\left(B_{i} a_{i}-\arctan \left(B_{i} a_{i}\right)\right)\right)\right) .
\end{aligned}
$$

In this model,

$$
\begin{aligned}
& B_{i}=\frac{a_{3} \cdot \sin \left(a_{4} \cdot\left(\arctan \left(a_{5} \cdot F_{Z i}\right)\right)\right)}{C_{i} \cdot D_{i}}, \\
& C_{i}=1.3, \\
& D_{i}=a_{1} \cdot F_{Z i}{ }^{2}+a_{2} \cdot F_{Z i}, \\
& E_{i}=a_{6} \cdot F_{Z i}{ }^{2}+a_{7} \cdot F_{Z i}+a_{8},
\end{aligned}
$$

$$
(i=1,2,3,4) \text {, }
$$

where $C_{1 f}$ and $C_{3 f}$ are the fitting coefficients of the tire, $\alpha_{i}(i=1,2,3,4$ denote the first bridge right wheel, the first bridge left wheel, the second bridge right wheel, and the second bridge left wheel, resp.) is the side-slip angle of the tire; $B_{i}, C_{i}, D_{i}$, and $E_{i}$ denote the stiffness, shape, crest, and curvature factors, respectively; $a_{1}, a_{2}, a_{3}, a_{4}, a_{5}, a_{6}, a_{7}$, and $a_{8}$ are obtained by test fitting; $F_{Z i}$ is the vertical forces on the tires. For details see appendix about $F_{Z i}, C_{1 f}$, and $C_{3 f}$. The required values $[26,27]$ are shown in Table 1 .

The relationship curve between the tire force $F_{i}$ and the side-slip angle $\alpha_{i}$ is shown in Figure 4.

The following are the tire rolling nonholonomic constraint equations [28], namely, the relationship between slip angle and the shimmy angle,

$$
\dot{\alpha}_{i}+\frac{v}{\sigma} \alpha_{i}+\frac{v}{\sigma} \theta_{i}-\frac{e_{i}}{\sigma} \dot{\theta}_{i}, \quad i=1,2,3,4 .
$$

2.4. Dry Friction Model Selection. In this study, the dry frictions in the suspension and steering mechanism ball kinematic pairs and steering gear kinematic pairs are considered. Suspension and steering systems are complex self-excited shimmy systems; thus, we made the kinematic pairs dry friction of the suspension and the steering system, except for the dry friction in the kingpin, equivalent to that of the kingpin because the dry friction in the kingpin is the main component of sports vice dry friction. We studied only the effect of the value of dry friction torque on the Hopf bifurcation of the shimmy system and therefore we change only the dry friction torque $M$, when making numerical calculations.

We selected the Coulomb model as the ideal friction model in this study. It has a constant value and is always in the opposite direction of the relative motion. This kind of friction always impedes movement and is always in the opposite direction of the movement speed. Its mathematical expression is

$$
\begin{aligned}
& M_{i j}=M \operatorname{sgn}\left(\dot{\theta}_{i j}\right) \quad(i, j=1,2), \\
& |M|=\left\{\begin{array}{l}
\mu_{s} N r\left(\dot{\theta}_{i j}=0\right) \\
\mu N r\left(\dot{\theta}_{i j} \neq 0\right)
\end{array} \quad(i, j=1,2),\right.
\end{aligned}
$$

where $\mu$ is the coefficient of sliding friction, $\mu_{s}$ is the coefficient of static friction, $N$ denotes a positive pressure, and $r$ is the main pin radius.

The parameter values of the sample vehicle are shown in Table 2 .

\section{Hopf Bifurcation Qualitative Analysis of the Shimmy System}

3.1. Existence Analysis of Hopf Bifurcation. The existence analysis and stability assessment of Hopf bifurcation in a nonlinear system are the most fundamental and important assessments in studying the dynamics of a nonlinear system. Hurwitz criterion is applied to investigate the existence of Hopf bifurcation in the dual-front axle shimmy system. The center manifold approach is applied to reduce and simplify the nonlinear system and obtain a two-dimensional system. The Hopf bifurcation paradigm is then applied to investigate the stability of the bifurcation of limit cycles.

Make $x_{1}=\theta_{1}, x_{2}=\dot{\theta}_{1}, x_{3}=\theta_{2}, x_{4}=\dot{\theta}_{2}, x_{5}=\varphi_{1}, x_{6}=\dot{\varphi}_{1}$, $x_{7}=\theta_{3}, x_{8}=\dot{\theta}_{3}, x_{9}=\theta_{4}, x_{10}=\dot{\theta}_{4}, x_{11}=\varphi_{2}, x_{12}=\dot{\varphi}_{2}$, $x_{13}=\delta_{1}, x_{14}=\dot{\delta}_{1}, x_{15}=\delta_{2}, x_{16}=\dot{\delta}_{2}, x_{17}=\delta_{3}, x_{18}=\dot{\delta}_{3}$, $x_{19}=\alpha_{1}, x_{20}=\alpha_{2}, x_{21}=\alpha_{3}$, and $x_{22}=\alpha_{4}$. Assume $X=$ $\left(x_{1}, x_{2}, x_{3}, x_{4}, x_{5}, x_{6}, x_{7}, x_{8}, x_{9}, x_{10}, x_{11}, x_{12}, x_{13}, x_{14}, x_{15}, x_{16}\right.$, $\left.x_{17}, x_{18}, x_{19}, x_{20}, x_{21}, x_{22}\right)$; according to the theory of nonlinear dynamics, the equilibrium point $X_{0}$ is obtained by solving the system static equations. Equations (6) to (14) can be expressed as the following static equations:

$$
\dot{X}=A X+F(X), \quad X \in R^{22},
$$

where $A$ is the Jacobian matrix in the equilibrium point vicinity of the system and $F(X)$ contains quadratic and cubic nonlinear terms.

According to Hurwitz criterion, the equilibrium point of the system is stable when $v=v_{1}$ or when $v=v_{2}$, whereas the equilibrium point of the system is unstable when $v_{1} \leq$ $v \leq v_{2}$ and when the critical speeds are $v_{1}=15.6 \mathrm{~km} / \mathrm{h}$ and $v_{2}=40.8 \mathrm{~km} / \mathrm{h}$. The eigenvalues of the Jacobian matrix $A$ are shown in Table 3.

According to Table 3, the Jacobian matrix $A$ of the system has a pair of purely imaginary eigenvalues when $v=v_{1}$ and $v=v_{2}$, and the other eigenvalues have negative real parts. 
TABLE 2: Sample car parameter values.

\begin{tabular}{|c|c|}
\hline Parameter & Value \\
\hline$\overline{I_{1}, I_{2} / \mathrm{kg} \cdot \mathrm{m}^{2} \cdot \mathrm{rad}^{-1}}$ & 16.8 \\
\hline$J_{1}, J_{2} / \mathrm{kg} \cdot \mathrm{m}^{2} \cdot \operatorname{rad}^{-1}$ & 210.8 \\
\hline$I_{c 1} / \mathrm{kg} \cdot \mathrm{m}^{2} \cdot \mathrm{rad}^{-1}$ & 0.38 \\
\hline$I_{c 2} / \mathrm{kg} \cdot \mathrm{m}^{2} \cdot \mathrm{rad}^{-1}$ & 0.22 \\
\hline$I_{c 3} / \mathrm{kg} \cdot \mathrm{m}^{2} \cdot \mathrm{rad}^{-1}$ & 0.28 \\
\hline$i_{k 1}, i_{k 2} / \mathrm{kg} \cdot \mathrm{m}^{2} \cdot \mathrm{rad}^{-1}$ & 14.2 \\
\hline$k_{h 1}, k_{h 2} / \mathrm{N} \cdot \mathrm{rad}^{-1}$ & 37790 \\
\hline$c_{h 1}, c_{h 2} / \mathrm{N} \cdot \mathrm{m} \cdot \mathrm{s} \cdot \mathrm{rad}^{-1}$ & 25 \\
\hline$c_{l 1}, c_{l 2} / \mathrm{N} \cdot \mathrm{m} \cdot \mathrm{s} \cdot \mathrm{rad}^{-1}$ & 42 \\
\hline$k_{n} / \mathrm{N} \cdot \mathrm{rad}^{-1}$ & 31360 \\
\hline$c_{n} / \mathrm{N} \cdot \mathrm{m} \cdot \mathrm{s} \cdot \mathrm{rad}^{-1}$ & 1027 \\
\hline$\gamma_{1}, \gamma_{2} /^{\circ}$ & 2.5 \\
\hline$l_{1}, l_{2} / \mathrm{m}$ & 0.07 \\
\hline$r_{1} / \mathrm{m}$ & 0.34 \\
\hline$r_{3} / \mathrm{m}$ & 0.47 \\
\hline$g_{2} / \mathrm{m}$ & 0.26 \\
\hline$a_{1} / \mathrm{m}$ & 0.21 \\
\hline$k_{z 1}, k_{z 2} / \mathrm{N} \cdot \mathrm{rad}^{-1}$ & 36000 \\
\hline$c_{z 1}, c_{z 2} / \mathrm{N} \cdot \mathrm{m} \cdot \mathrm{s} \cdot \mathrm{rad}^{-1}$ & 30 \\
\hline$k_{t 1}, k_{t 2} / \mathrm{N} \cdot \mathrm{m}^{-1}$ & 37200 \\
\hline$c_{t 1}, c_{t 2} / \mathrm{N} \cdot \mathrm{s}$ & 15 \\
\hline$k_{c} / \mathrm{N} \cdot \mathrm{rad}^{-1}$ & 1060000 \\
\hline$f$ & 0.015 \\
\hline$k_{t} / \mathrm{N} \cdot \mathrm{rad}^{-1}$ & 31000 \\
\hline$c_{t} / \mathrm{N} \cdot \mathrm{m} \cdot \mathrm{s} \cdot \mathrm{rad}^{-1}$ & 10 \\
\hline$\rho / \mathrm{N} \cdot \mathrm{rad}^{-1}$ & 765000 \\
\hline$R / \mathrm{m}$ & 0.51 \\
\hline$e_{1}, e_{2} / \mathrm{m}$ & 0.07 \\
\hline$h_{1}, h_{2} / \mathrm{m}$ & 0.45 \\
\hline$B_{1}, B_{2} / \mathrm{m}$ & 2.06 \\
\hline$r_{2} / \mathrm{m}$ & 0.42 \\
\hline$g_{1} / \mathrm{m}$ & 0.17 \\
\hline$g_{3} / \mathrm{m}$ & 0.22 \\
\hline$a_{2} / \mathrm{m}$ & 0.21 \\
\hline
\end{tabular}

Using the Hopf bifurcation theorem as basis, we can conclude that the critical speeds $v_{1}=15.6 \mathrm{~km} / \mathrm{h}$ and $v_{2}=40.8 \mathrm{~km} / \mathrm{h}$ are the bifurcation points of the systems, and a two-dimensional center manifold exists. When $v_{1} \leq v \leq v_{2}$, positive real part eigenvalues exist, and the original shimmy system has selfexcited vibrations and produces limit cycles. When $v<v_{1}$ or $v>v_{2}$, all the eigenvalues of $A$ have negative real parts, and the original shimmy system is stable asymptotically and eventually becomes balanced.

3.2. Stability Analysis of Limit Cycles. The center manifold approach is applied to determine the stability of the original system in low-dimensional systems and to reduce the original system. Assuming $\mu_{k}=v-v_{k}(k=1,2), \mu_{k}$ is an increment in the speed bifurcation parameter at the bifurcation point and has a minimum value. Using the nonsingular transformation $X=P Y$, where $Y$ has the same dimensions as $X, P$
TABLE 3: Eigenvalues of Jacobian matrix $A$ corresponding to the critical speed.

\begin{tabular}{lccc}
\hline \multicolumn{2}{c}{$v_{1}$ corresponding eigenvalues } & \multicolumn{2}{c}{$v_{2}$ corresponding eigenvalues } \\
\hline$\lambda_{1}$ & $0+12.11 i$ & $\lambda_{1}$ & $0+14.56 i$ \\
$\lambda_{2}$ & $0-12.11 i$ & $\lambda_{2}$ & $0-14.56 i$ \\
$\lambda_{3}$ & $-165.44+780.83 i$ & $\lambda_{3}$ & $-165.44+780.83 i$ \\
$\lambda_{4}$ & $-165.44-780.83 i$ & $\lambda_{4}$ & $-165.44-780.83 i$ \\
$\lambda_{5}$ & $-71.40+549.08 i$ & $\lambda_{5}$ & $-71.40+549.08 i$ \\
$\lambda_{6}$ & $-71.40-549.08 i$ & $\lambda_{6}$ & $-71.40-549.08 i$ \\
$\lambda_{7}$ & $-4.37+63.15 i$ & $\lambda_{7}$ & $-4.25+63.16 i$ \\
$\lambda_{8}$ & $-4.37-63.15 i$ & $\lambda_{8}$ & $-4.25-63.16 i$ \\
$\lambda_{9}$ & $-4.34+61.78 i$ & $\lambda_{9}$ & $-4.22+61.79 i$ \\
$\lambda_{10}$ & $-4.34-61.78 i$ & $\lambda_{10}$ & $-4.22-61.79 i$ \\
$\lambda_{11}$ & $-2.18+26.88 i$ & $\lambda_{11}$ & $-1.62+27.73 i$ \\
$\lambda_{12}$ & $-2.18-26.88 i$ & $\lambda_{12}$ & $-1.62-27.73 i$ \\
$\lambda_{13}$ & $-2.24+26.81 i$ & $\lambda_{13}$ & $-1.79+27.46 i$ \\
$\lambda_{14}$ & $-2.24-26.81 i$ & $\lambda_{14}$ & $-1.79-27.46 i$ \\
$\lambda_{15}$ & $-1.22+16.21 i$ & $\lambda_{15}$ & $-0.98+17.65 i$ \\
$\lambda_{16}$ & $-1.22-16.21 i$ & $\lambda_{16}$ & $-0.98-17.65 i$ \\
$\lambda_{17}$ & $-243.78+911.55 i$ & $\lambda_{17}$ & $-243.78+911.55 i$ \\
$\lambda_{18}$ & $-243.78-911.55 i$ & $\lambda_{18}$ & $-243.78-911.55 i$ \\
$\lambda_{19}$ & -8.92 & $\lambda_{19}$ & -15.14 \\
$\lambda_{20}$ & -6.64 & $\lambda_{20}$ & -13.58 \\
$\lambda_{21}$ & -3.55 & $\lambda_{21}$ & -9.09 \\
$\lambda_{22}$ & -3.54 & $\lambda_{22}$ & -9.10 \\
\hline & & &
\end{tabular}

is composed of the real and imaginary parts of all the eigenvectors, and $\Lambda=P^{-1} A P$ is $22 \times 22$ diagonal matrix; (20) is converted into

$$
\dot{Y}=\Lambda Y+P^{-1} g\left(Y, \mu_{k}\right) .
$$

Using center manifold theory to reduce dimension, the center manifold $W^{c}$ of the expansion system represented by (21) is tangent to the plane of $\left(y_{1}, y_{2}, \mu_{k}\right)$ at the singular point $\left(X_{0}, v_{0}\right)$, assuming the center manifold

$$
\begin{aligned}
y_{i}= & h_{i}\left(y_{1}, y_{2}, \mu_{k}\right) \\
= & h_{i 1} y_{1}^{2}+h_{i 2} \mu_{k} y_{1}+h_{i 3} \mu_{k} y_{2}+h_{i 4} y_{2}^{2}, \\
& i=3,4, \ldots, 21,22, \\
\dot{y}_{i}= & \frac{\partial h_{i}\left(y_{1}, y_{2}, \mu_{k}\right)}{\partial y_{1}} \dot{y}_{1}+\frac{\partial h_{i}\left(y_{1}, y_{2}, \mu_{k}\right)}{\partial y_{2}} \dot{y}_{2} \\
& +\frac{\partial h_{i}\left(y_{1}, y_{2}, \mu_{k}\right)}{\partial \mu_{k}} \mu_{k} .
\end{aligned}
$$

Equation (22) is substituted into (21) and is then combined with (23), after which the coefficients of the same item on both sides of the equation are compared using the software Maple. In solving the linear equations, the coefficients of $h_{i}\left(y_{1}, y_{2}, v\right)(i=3,4, \ldots, 21,22)$ can be obtained and brought into the previous two equations of (21). After simplifying 
the equation, reduction equations can be obtained when $v_{1}=$ $15.6 \mathrm{~km} / \mathrm{h}$ or $v_{2}=40.8 \mathrm{~km} / \mathrm{h}$ at the center manifold.

When $v=v_{1}=15.6 \mathrm{~km} / \mathrm{h}$, the reduction equation is

$$
\begin{aligned}
\dot{y}_{1}= & 1.21 \times 10 y_{2}+0.762 y_{2} \mu_{1}-0.396 y_{1} \mu_{1}-0.169 \\
& \times 10^{-3} y_{2} \mu_{1}^{2}-0.156 \times 10^{-4} y_{2}^{3}-0.118 \\
& \times 10^{-3} y_{1} \mu_{1}^{2}-0.556 \times 10^{-3} y_{1} y_{2}^{2}-0.66 \\
& \times 10^{-2} y_{1}^{2} y_{2}-0.261 \times 10^{-1} y_{1}^{3}+0.426 \\
& \times 10^{-4} y_{1}^{2} y_{2} \mu_{1}+0.464 \times 10^{-4} y_{1}^{3} \mu_{1}, \\
\dot{y}_{2}= & -1.21 \times 10 y_{1}-0.148 y_{1} \mu_{1}+0.853 y_{2} \mu_{1}+0.998 \\
& \times 10^{-4} y_{1} \mu_{1}^{2}+0.113 \times 10^{-1} y_{1}^{3}+0.429 \\
& \times 10^{-4} y_{2} \mu_{1}^{2}+0.286 \times 10^{-2} y_{1}^{2} y_{2}+0.242 \\
& \times 10^{-3} y_{1} y_{2}^{2}-0.201 \times 10^{-4} y_{1}^{3} \mu_{1}-0.187 \\
& \times 10^{-4} y_{1}^{2} y_{2} \mu_{1} .
\end{aligned}
$$

When $v=v_{2}=40.8 \mathrm{~km} / \mathrm{h}$, the reduction equation is

$$
\begin{aligned}
\dot{y}_{1}= & 1.46 \times 10 y_{2}-0.414 \times 10^{-2} y_{1}+0.522 y_{2} \mu_{2} \\
& -0.550 y_{1} \mu_{2}-0.784 \times 10^{-2} y_{2}^{3}+0.118 \\
& \times 10^{-4} y_{1} \mu_{2}^{2}-0.487 \times 10^{-1} y_{2}^{2} y_{1} \\
& -0.101 y_{2} y_{1}^{2}-0.697 \times 10^{-1} y_{1}^{3}-0.259 \\
& \times 10^{-4} y_{2} y_{1}^{2} \mu_{2}-0.231 \times 10^{-4} y_{1}^{3} \mu_{2}, \\
\dot{y}_{2}= & -1.46 \times 10 y_{1}-0.414 \times 10^{-2} y_{2}+0.275 \\
& \times 10^{-1} y_{1} \mu_{2}+0.261 y_{2} \mu_{2}+0.381 \\
& \times 10^{-4} y_{1} \mu_{2}^{2}+0.391 \times 10^{-1} y_{1}^{3}-0.531 \\
& \times 10^{-4} y_{2} \mu_{2}^{2}+0.565 \times 10^{-1} y_{2} y_{1}^{2}+0.273 \\
& \times 10^{-1} y_{2}^{2} y_{1}+0.439 \times 10^{-2} y_{2}^{3}+0.103 \\
& \times 10^{-4} y_{1}^{3} \mu_{2}+0.116 \times 10^{-4} y_{2} y_{1}^{2} \mu_{2} .
\end{aligned}
$$

According to the literature [29], the Hopf bifurcation paradigm under the polar coordinates of (24) to (25) can be obtained using Maple procedures:

$$
\begin{aligned}
& \dot{r}=\text { dur }+a r^{3}+\text { h.o.t., } \\
& \dot{\theta}=\omega+b r^{2}+\text { h.o.t., }
\end{aligned}
$$

where h.o.t. represents infinitesimals of higher order.

According to $(24) \sim(28)$, we can obtain the following.

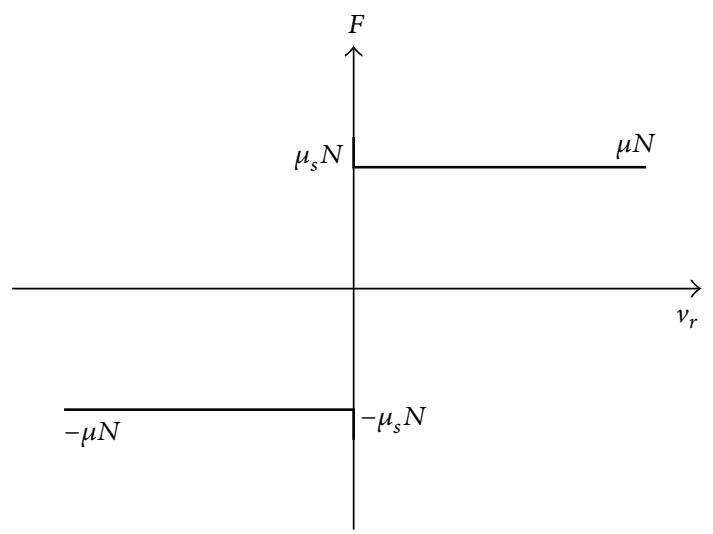

FIGURE 5: Shimmy system friction with the change of velocity curve.

$$
\begin{aligned}
& \text { For } v=v_{1}=15.6 \mathrm{~km} / \mathrm{h}, \\
& \begin{aligned}
\dot{r}= & \left(-0.751 \times 10^{-4} \mu_{1}+0.229\right) \mu_{1} r+(-0.193 \\
& \left.\times 10^{-6} \mu_{1}^{2}+0.236 \times 10^{-4} \mu_{1}-0.106 \times 10^{-1}\right) r^{3} \\
& + \text { h.o.t. }
\end{aligned}
\end{aligned}
$$

For $v=v_{2}=40.8 \mathrm{~km} / \mathrm{h}$,

$$
\begin{aligned}
\dot{r}= & \left(-0.413 \times 10^{-4} \mu_{2}-0.145\right) \mu_{2} r+(-0.495 \\
& \left.\times 10^{-6} \mu_{2}^{2}-0.133 \times 10^{-4} \mu_{2}-0.476 \times 10^{-1}\right) r^{3} \\
& + \text { h.o.t. }
\end{aligned}
$$

According to (27) and (28), we can obtain the bifurcation diagram at equilibrium point $X_{0}$ of each critical velocity. The diagram is shown in Figure 6.

According to Figure 6, Hopf bifurcations are supercritical at the critical speeds $v_{1}$ and $v_{2}$. When $v<v_{1}=15.6 \mathrm{~km} / \mathrm{h}$ (i.e., $\mu_{1}<0$ ), shimmy does not occur in the system; that is, the system is stable and equilibrium point $X_{0}$ is a stable focus. When $v_{1}<v<v_{2}$ (i.e., $\mu_{1}>0$ and $\mu_{2}<0$ ), shimmy occurs in the system, equilibrium point $X_{0}$ is an unstable focus, and a stable limit cycle appears. The phenomenon of the mutation of a stable focus into a stable limit cycle is called the limit cycle hard to produce or stable hard loss. When $v>v_{2}=40.8 \mathrm{~km} / \mathrm{h}$ (i.e., $\mu_{2}>0$ ), the equilibrium point turns into a stable focus again, the limit cycle disappears, the shimmy phenomenon disappears, and the system tends to be stable. Thus, dual-front axle shimmy is a typical self-excited vibration.

\section{Calculation and Analysis of the Hopf Bifurcation in the Shimmy System}

4.1. Numerical Calculation and Analysis. Using the motion equations of the sample vehicle shimmy system and the Runge-Kutta method for the numerical calculation of shimmy systems, the bifurcation diagrams in Figure 7 show that the left wheel swing angles of the first and second bridges vary with speed. 


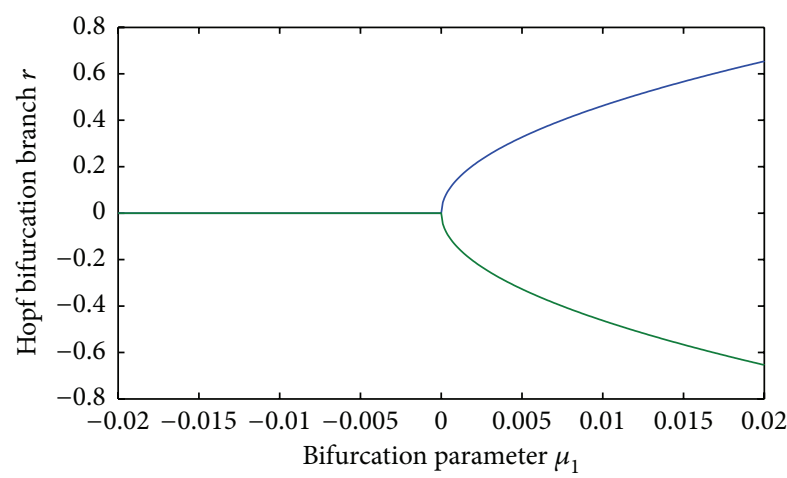

(a) Bifurcation diagram at $v_{1}$

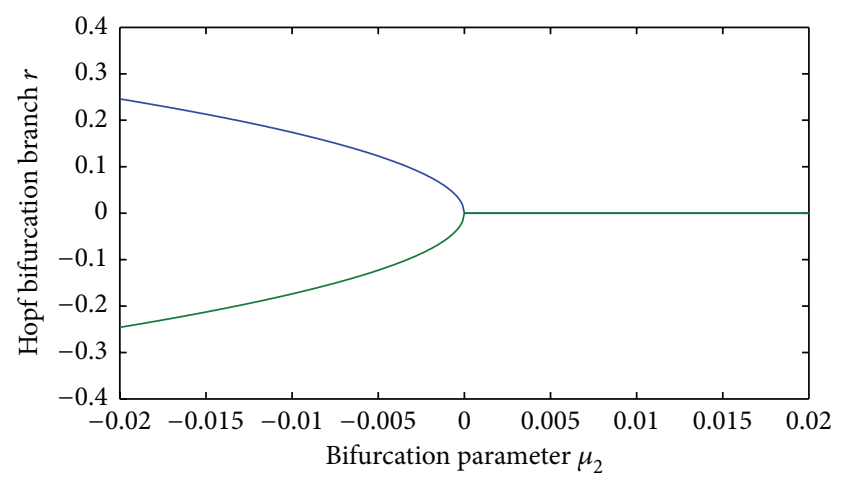

(b) Bifurcation diagram at $v_{2}$

FIgURE 6: Bifurcation diagram of $X_{0}$ at two critical speeds.

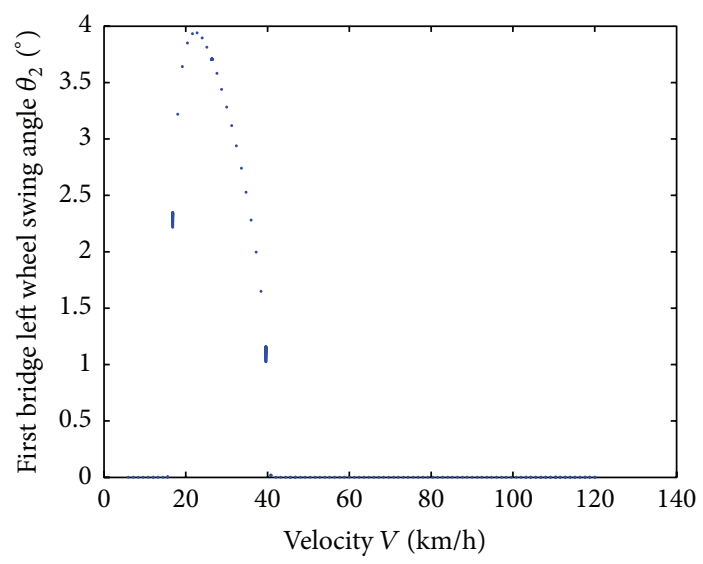

(a) First bridge left wheel

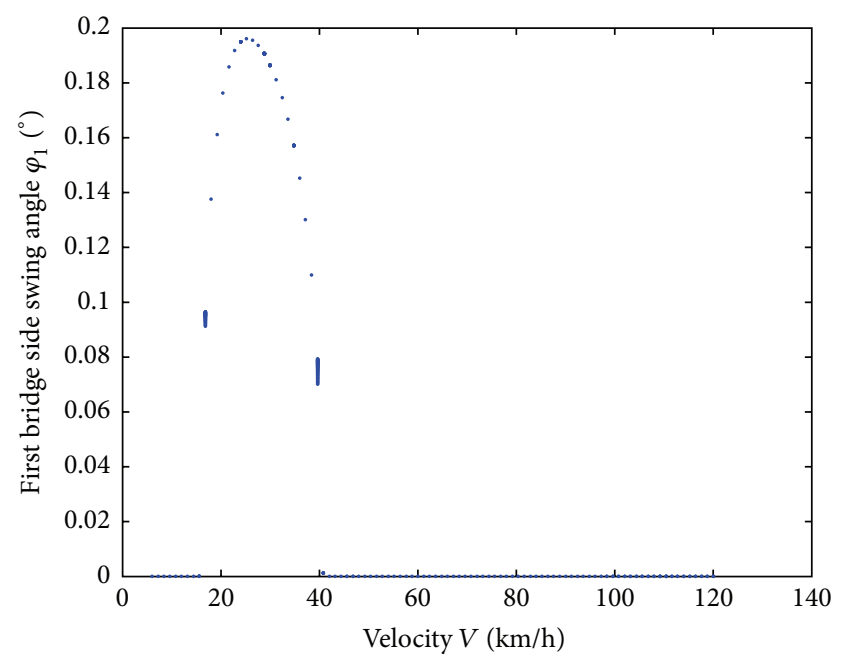

(c) The first bridge side pendulum

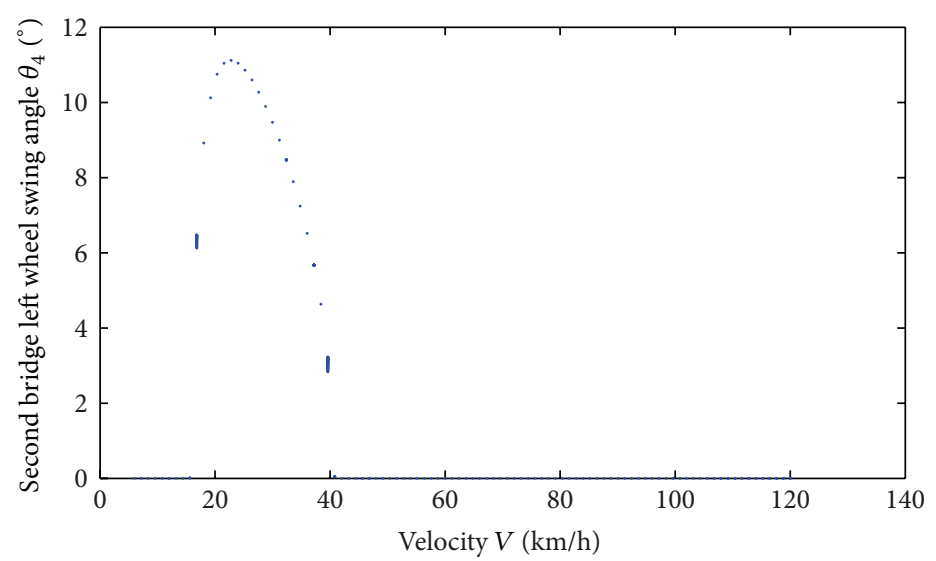

(b) Second bridge left wheel

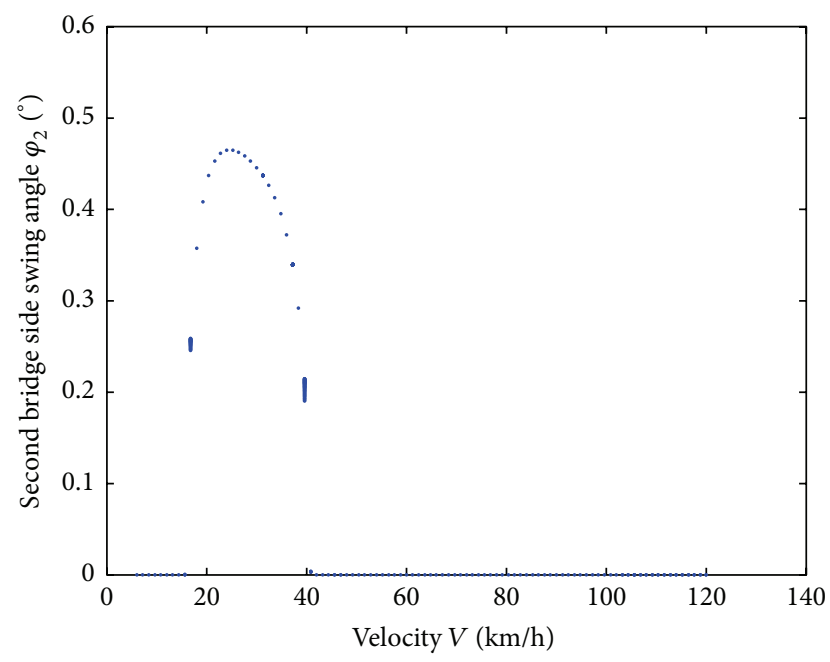

(d) The second bridge side pendulum

Figure 7: First and second bridge Hopf bifurcation with change of speed.

According to Figure 7, when $v<15.6 \mathrm{~km} / \mathrm{h}$ and $v>$ $40.8 \mathrm{~km} / \mathrm{h}$, the vibration of a dual-front axle system gradually stabilizes, whereas when $15.6 \mathrm{~km} / \mathrm{h}<v<40.8 \mathrm{~km} / \mathrm{h}$ periodic oscillations occur in the dual-front axle steering system, resulting in the limit cycle. These results are consistent with the qualitative calculation results in Section 3.1, which in turn show that the theoretical qualitative methods are consistent with the numerical methods.

To better analyze the state changes in a dual-front axle system within the shimmy speed range, we provide a swing 


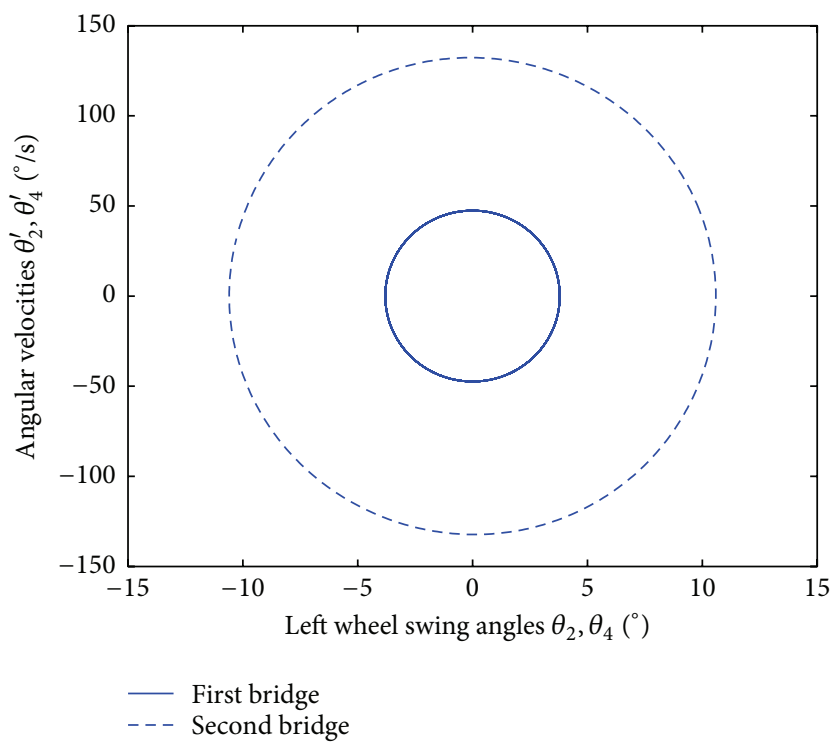

(a) $v=20 \mathrm{~km} / \mathrm{h}$

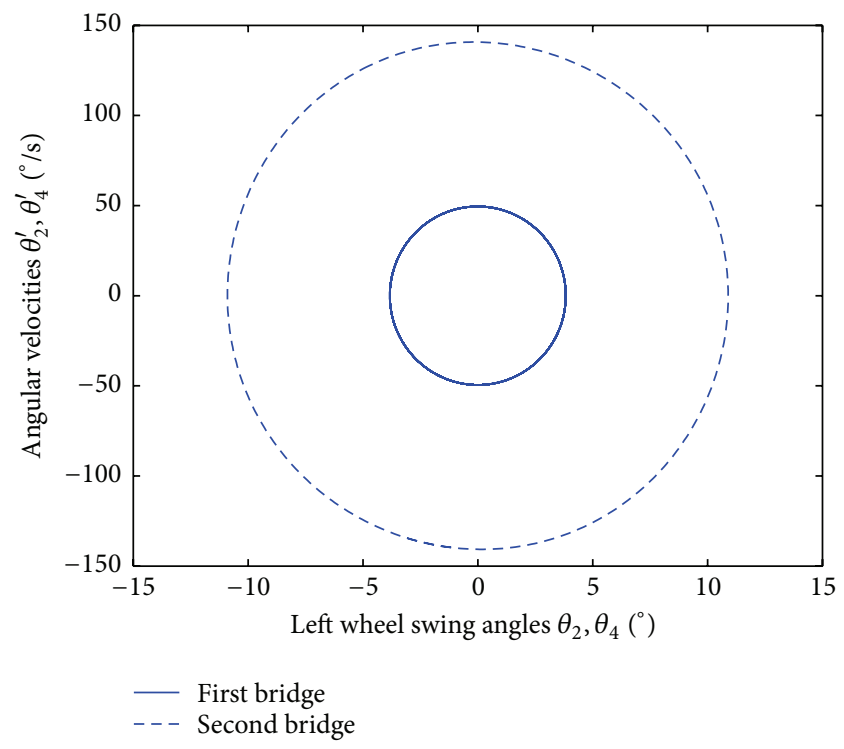

(b) $v=25 \mathrm{~km} / \mathrm{h}$

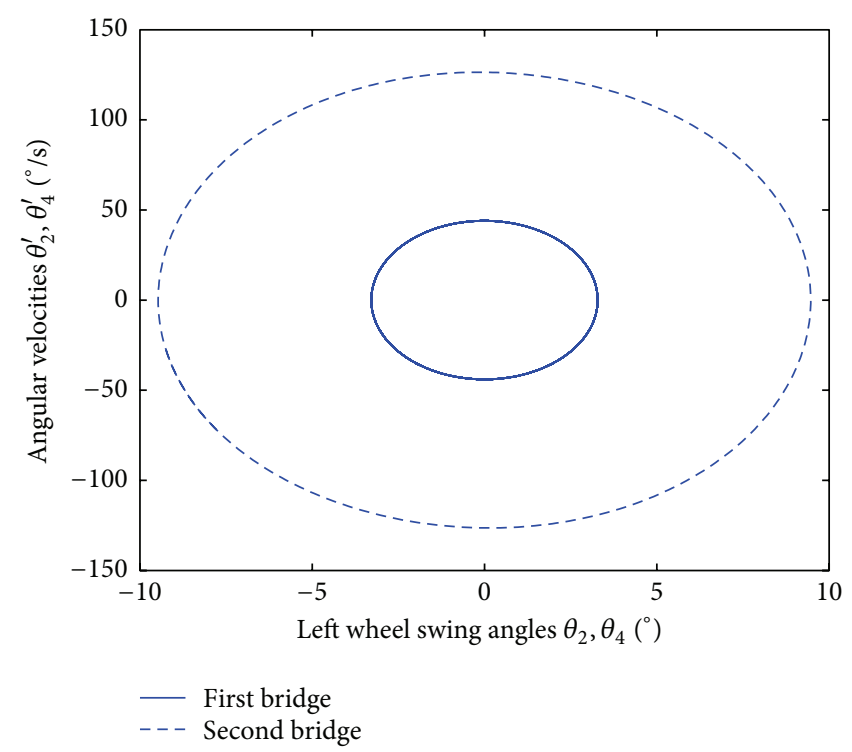

(c) $v=30 \mathrm{~km} / \mathrm{h}$

Figure 8: Phase diagram of the two front wheels at different speed.

angle phase diagram of the left wheel of the first and second bridges of heavy-duty vehicles under different speeds, as shown in Figure 8.

The conclusions on the shimmy characteristics and laws pertaining to the left wheel of the first and second bridges under different speeds, as shown in Figures 8(a)-8(c), are drawn. The results are summarized in Table 4.

(1) From Figures 8(a) to 8(c), in the bifurcation speed range, the shimmy of the steering wheel is the limit cycle vibration with a larger amplitude. When the speed increases, the self-excited oscillation amplitude of the swing angle increases initially and then decreases, and the amplitude of the swing angle acceleration has the same trend. The maximum amplitude
TABle 4: Shimmy characteristic at different speed.

\begin{tabular}{lccc}
\hline$v / \mathrm{km} \cdot \mathrm{h}^{-1}$ & 20 & 25 & 30 \\
$\theta_{2} /^{\circ}$ & 3.73 & 3.81 & 3.28 \\
$\theta_{4}{ }^{\circ}$ & 10.55 & 10.92 & 9.47 \\
$\Delta \theta /^{\circ}$ & 6.82 & 7.11 & 6.19 \\
$\dot{\theta}_{2} / \mathrm{rad} \cdot \mathrm{s}^{-1}$ & 0.83 & 0.86 & 0.76 \\
$\dot{\theta}_{4} / \mathrm{rad} \cdot \mathrm{s}^{-1}$ & 2.31 & 2.46 & 2.21 \\
$\Delta \dot{\theta} / \mathrm{rad} \cdot \mathrm{s}^{-1}$ & 1.48 & 1.59 & 1.44 \\
\hline
\end{tabular}

of the swing angle of the left wheel of the first bridge is $3.81^{\circ}$, and the maximum amplitude of the swing angle acceleration is $0.86 \mathrm{rad} / \mathrm{s}$. The maximum amplitude of the swing angle of the left wheel of the second bridge 


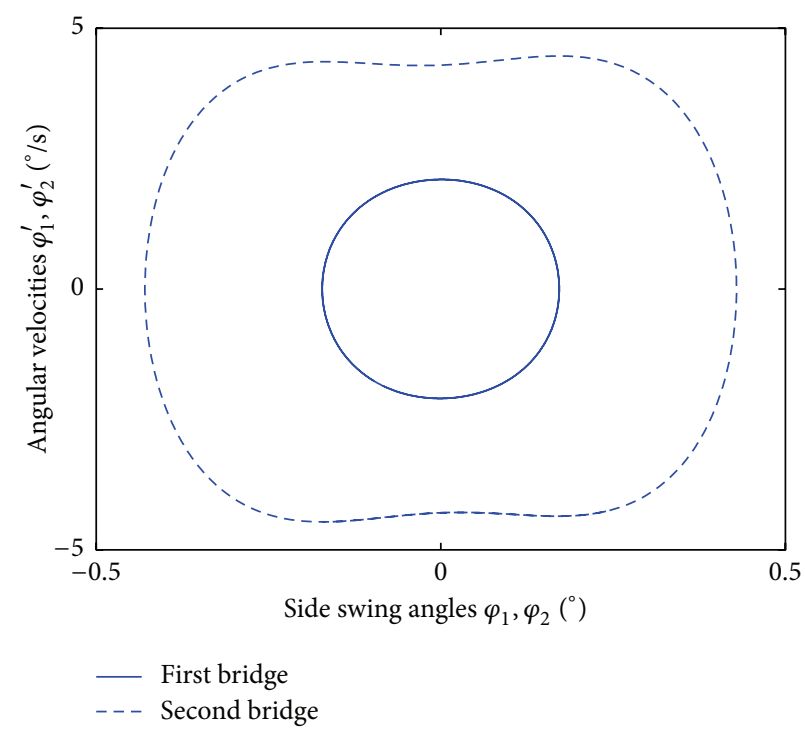

(a) $v=20 \mathrm{~km} / \mathrm{h}$

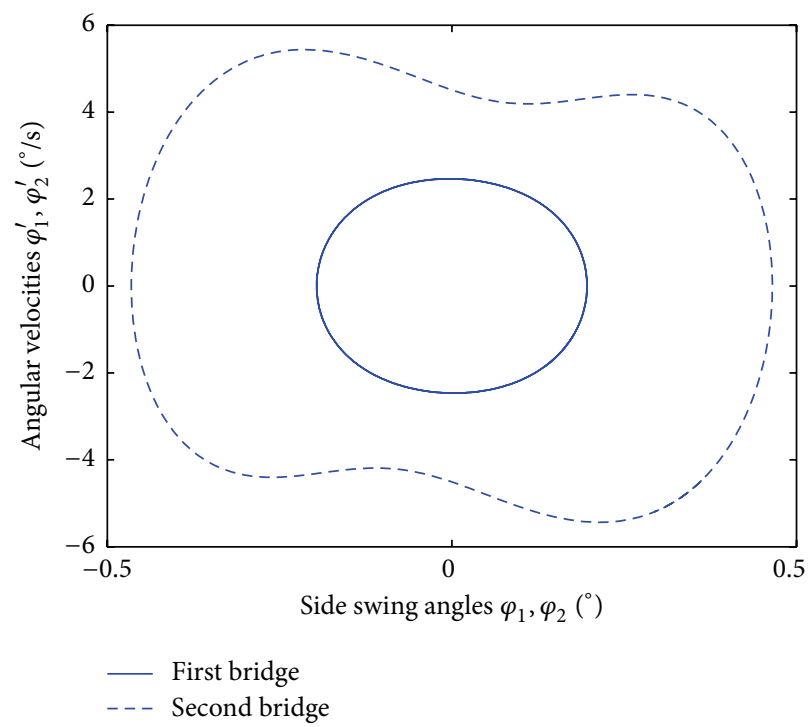

(b) $v=25 \mathrm{~km} / \mathrm{h}$

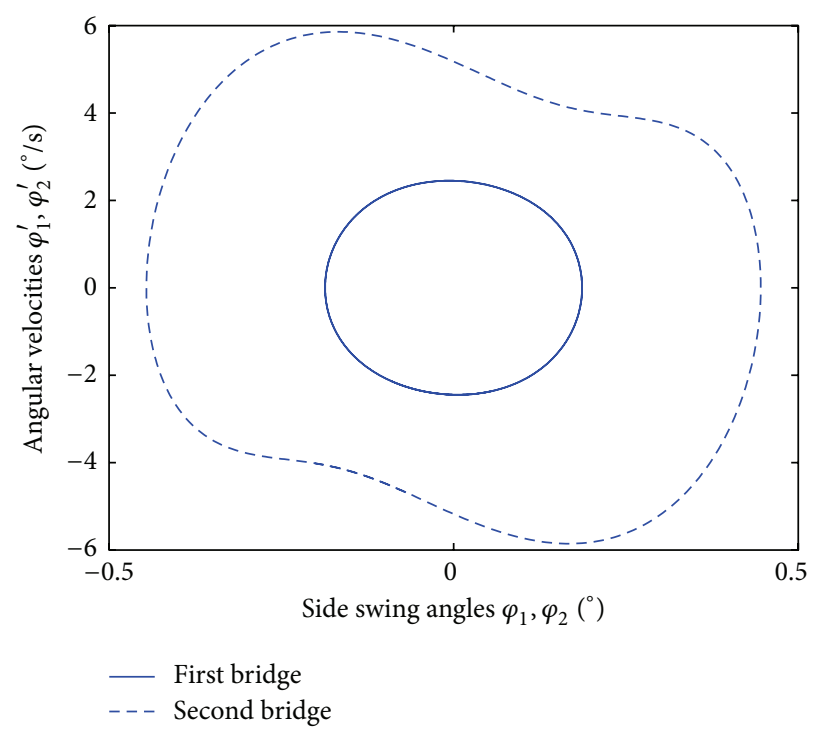

(c) $v=30 \mathrm{~km} / \mathrm{h}$

FIGURE 9: Phase diagram of the two bridges side pendulum at different speed.

is $10.92^{\circ}$, and the maximum amplitude of the swing angle acceleration is $2.46 \mathrm{rad} / \mathrm{s}$. This phenomenon leads the vehicle to hunting movement and makes the driver feel the tension and fatigue.

(2) According to Figures 8(a)-8(c) and Table 4, the variations in the left wheel swing angle differences and angular acceleration differences of the first and the second bridges are consistent with the variations in the swing angles and angular accelerations. The maximum difference between the left wheel swing angles of the two bridges is $7.11^{\circ}$, whereas the minimum difference is $6.19^{\circ}$. The maximum difference of the angular acceleration is $1.59 \mathrm{rad} / \mathrm{s}$, whereas the minimum difference is $1.44 \mathrm{rad} / \mathrm{s}$. These results show that the intensities of the tire shimmy of the first and second bridges are in a state of serious imbalance. The intensity of the tire shimmy at the second bridge is significantly greater than that at the first bridge during a shimmy. These results coincide with the actual use of heavy-duty trucks, in which the tire of the second bridge experiences more severe wear than the tire of the first bridge.

(3) According to Figures 8(a)-8(c) and Table 4, speed is an important bifurcation parameter in a dual-front axle shimmy system. Thus, driving speed should avoid the bifurcation range as much as possible.

Figure 9 shows the side pendulum phase diagrams of the first and second bridges of heavy-duty vehicles under different speeds. 


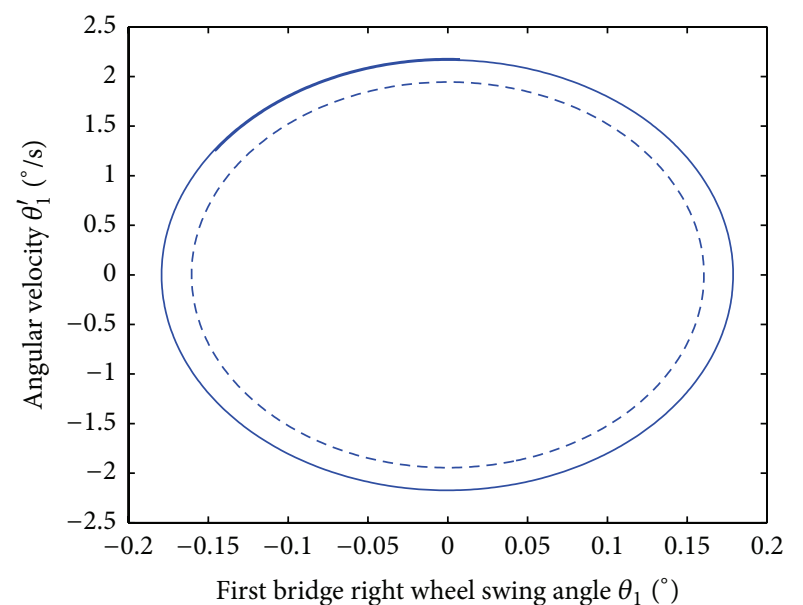

_ Original system

- - - Reduced system

(a) First bridge right wheel

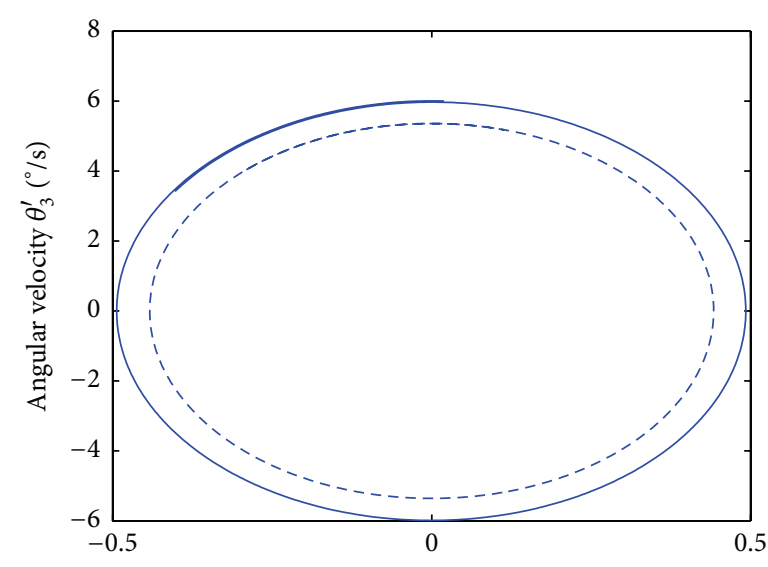

Second bridge right wheel swing angle $\theta_{3}\left({ }^{\circ}\right)$

— Original system

- - Reduced system

(c) Second bridge right wheel

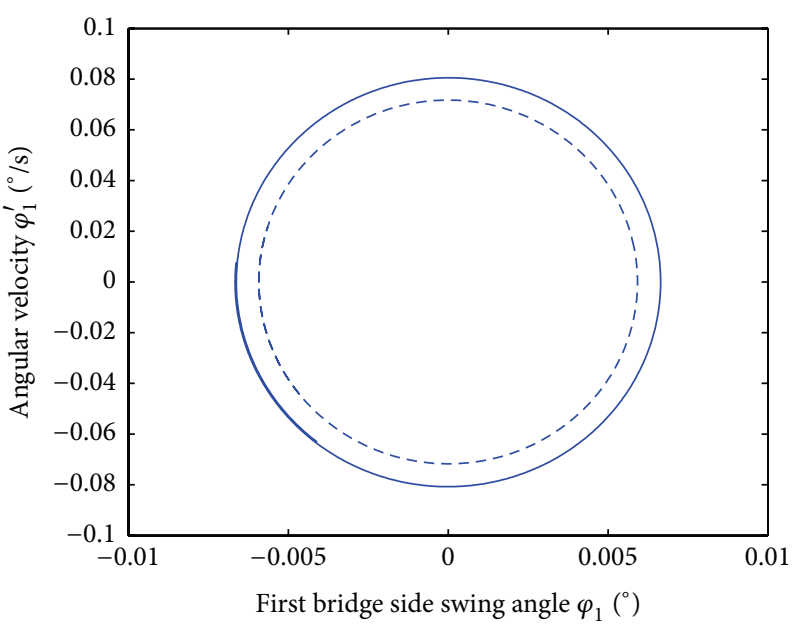

- Original system

- - - Reduced system

(e) The first bridge side pendulum

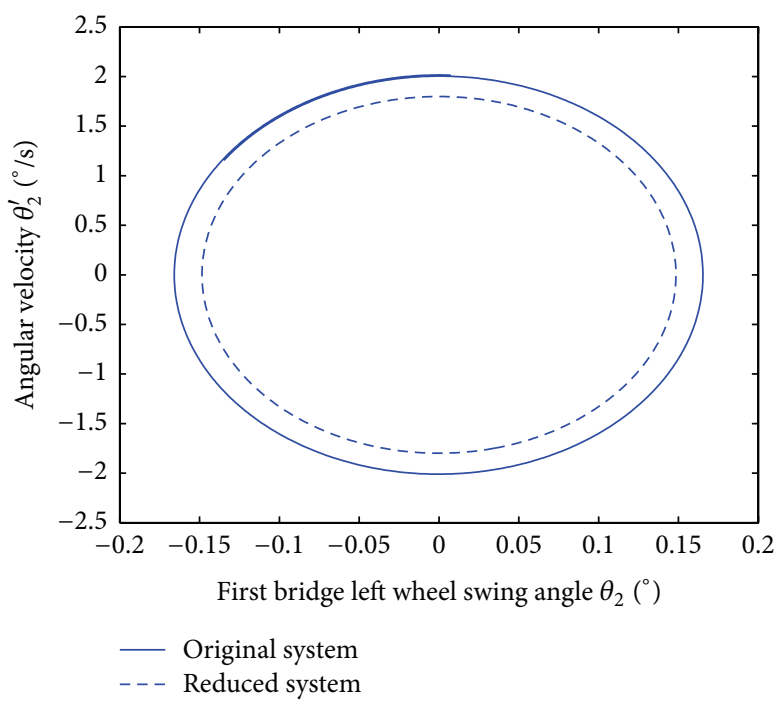

(b) First bridge left wheel

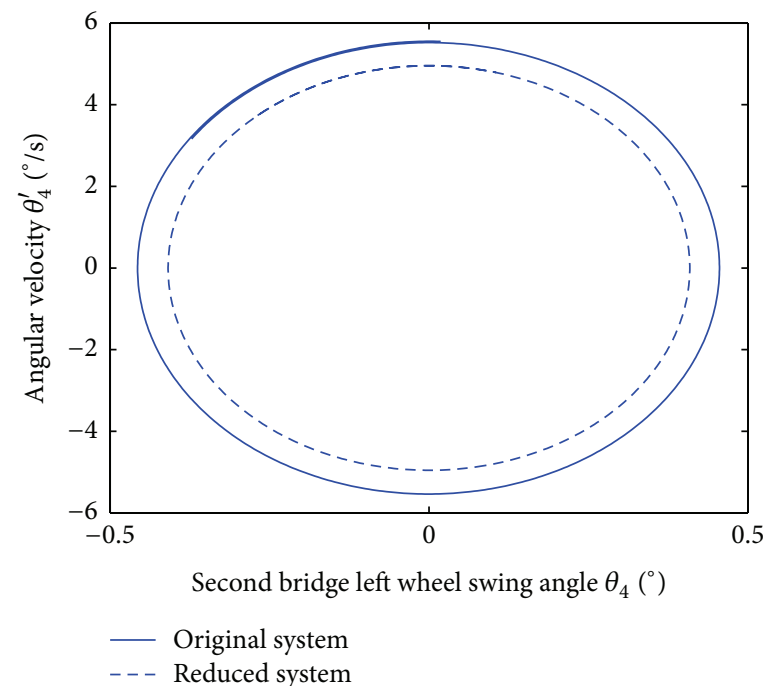

(d) Second bridge left wheel

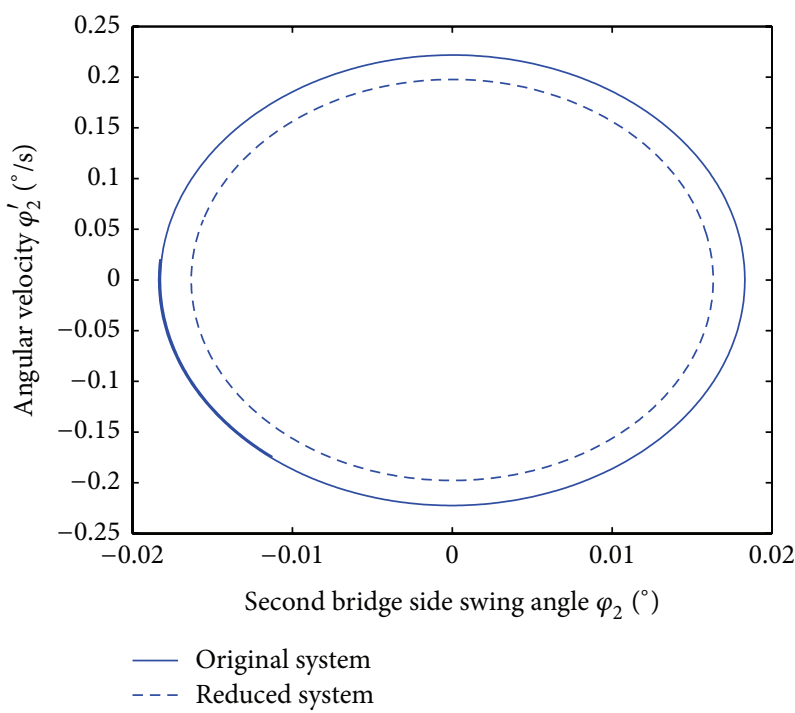

(f) The second bridge side pendulum

Figure 10: Continued. 


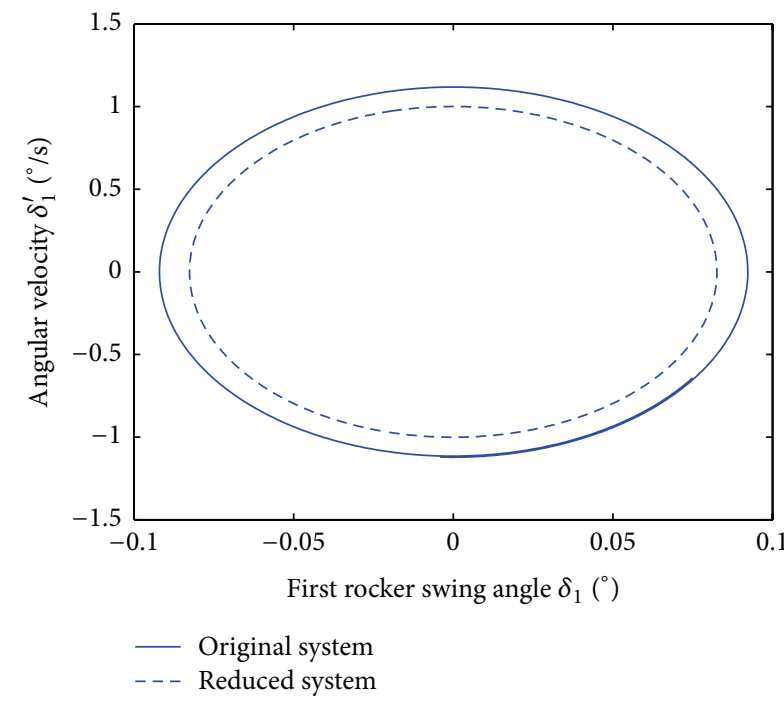

(g) First rocker swing angle

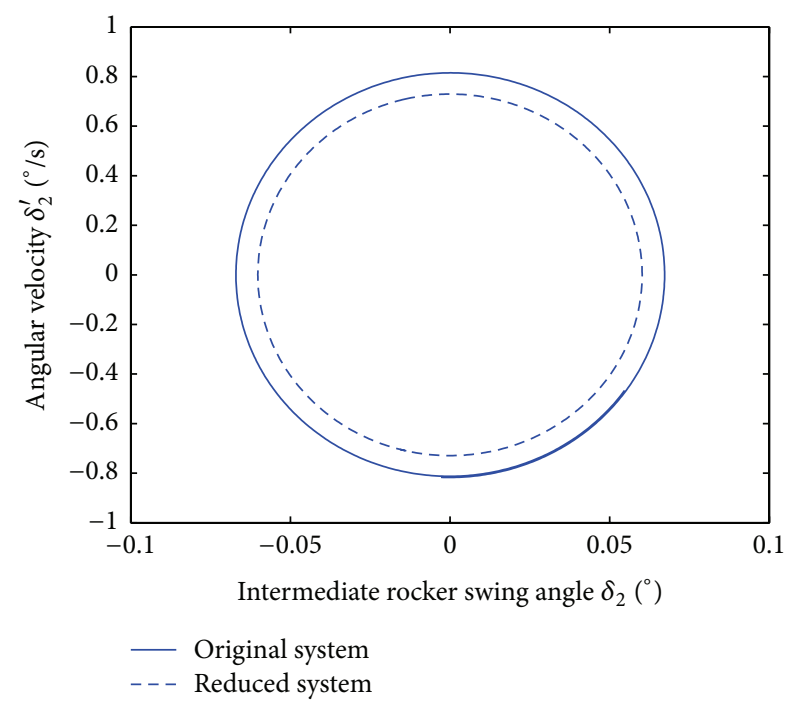

(h) Intermediate rocker swing angle

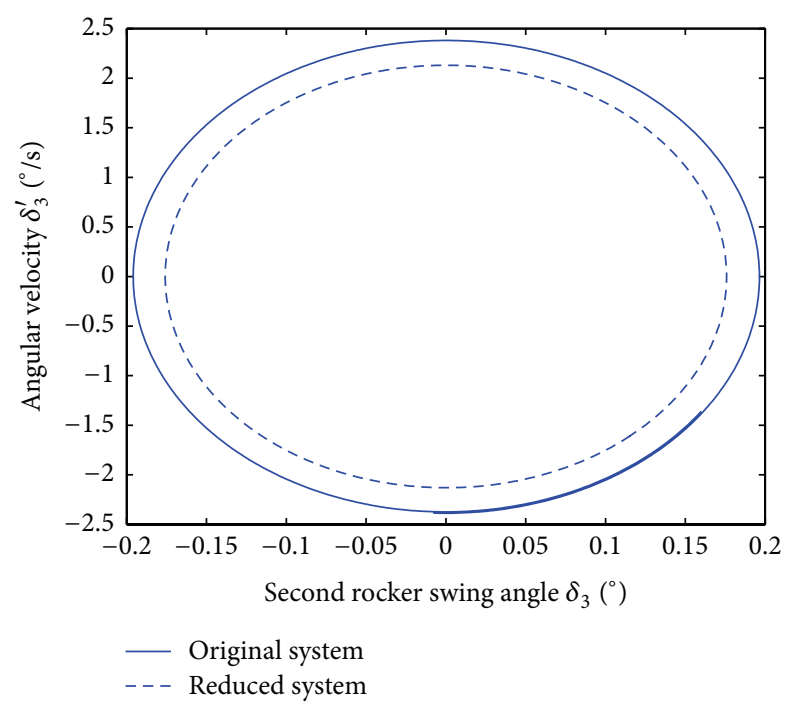

(i) Second rocker swing angle

FIGURE 10: For $v=15.6 \mathrm{~km} / \mathrm{h}$ comparison of limit cycle of original systems and limit cycle of dimensionality reduction system.

Figure 9 shows that the side pendulum phase diagram of the first bridge is elliptically regular, indicating that the first bridge side pendulum angle is at its maximum, when the side pendulum angular velocity is at its minimum, the side pendulum angle is at its minimum, and the side pendulum angular velocity is at its maximum. The pendulum phase diagram curve of the second bridge side is inwardly recessed along $y$-axis direction, indicating that the second bridge side pendulum angle is at its maximum, when the side pendulum angular velocity is at its minimum and the side pendulum angle is at its minimum, but the side pendulum angular velocity is not at its maximum. Taking Figure 9(b) as an example, when the angular velocity is less than 0 , the slope of the first bridge phase diagram trajectory only changes once from negative to positive. By contrast, the slope of the second bridge phase diagram trajectory changes twice from negative to positive and then becomes positive. Figure 9 shows that the value of the second bridge side pendulum is not only greater than that of the first bridge but also more complex than that of the first bridge.

\subsection{Dimensionality Reduction System Limit Cycle Compared} with the Original Systems. When $v=v_{1}=15.6 \mathrm{~km} / \mathrm{h}, \mu_{1}=$ $0.001 v_{1}$, we generate the original system limit cycle phase diagram with the use of the four- and five-order Runge-Kutta method and the dimensionality reduction system limit cycle phase diagram with the use of center manifold theory. These diagrams are shown in Figure 10.

When $v=v_{2}=40.8 \mathrm{~km} / \mathrm{h}, \mu_{2}=0.001 v_{2}$, we generate the original system limit cycle phase diagram with the use of the four- and five-order Runge-Kutta method and the dimensionality reduction system limit cycle phase diagram with the use of center manifold theory. These diagrams are shown in Figure 11. 


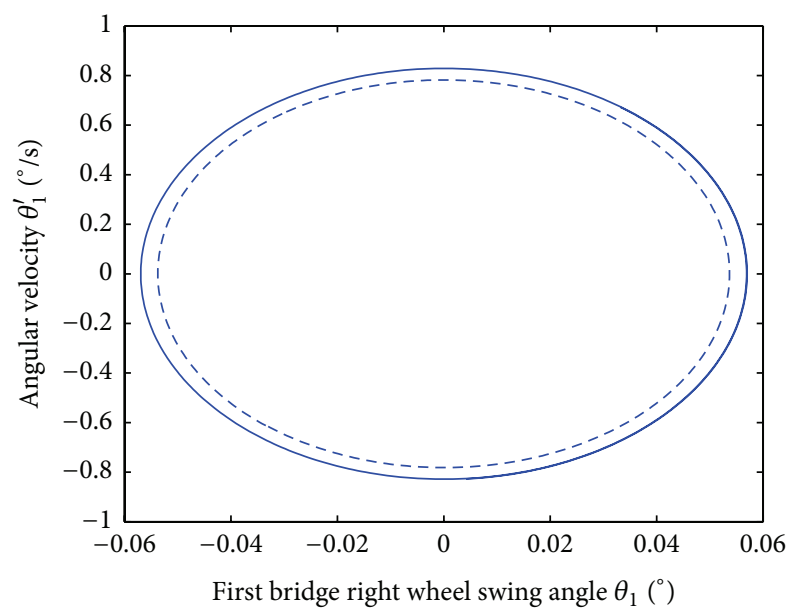

- Original system

- - - Reduced system

(a) First bridge right wheel

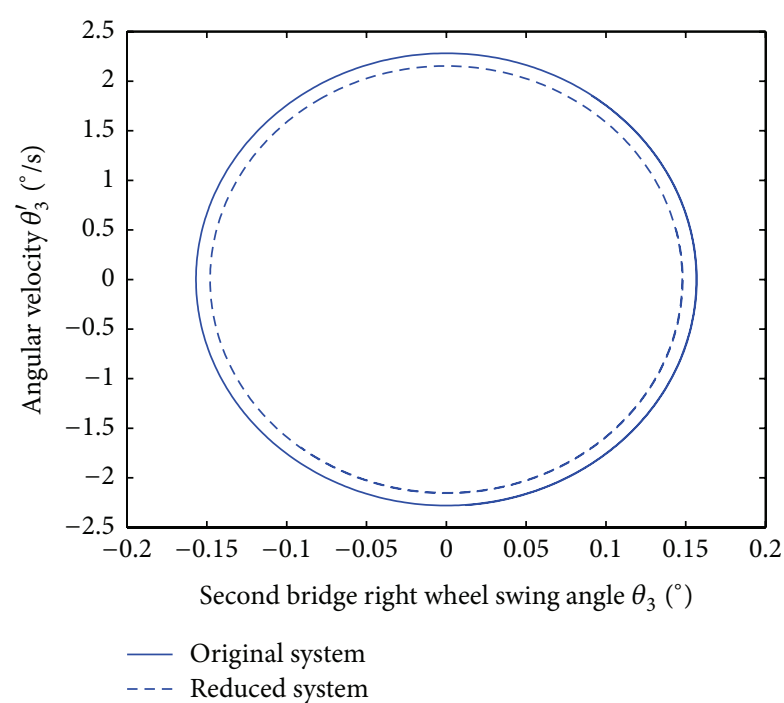

(c) Second bridge right wheel

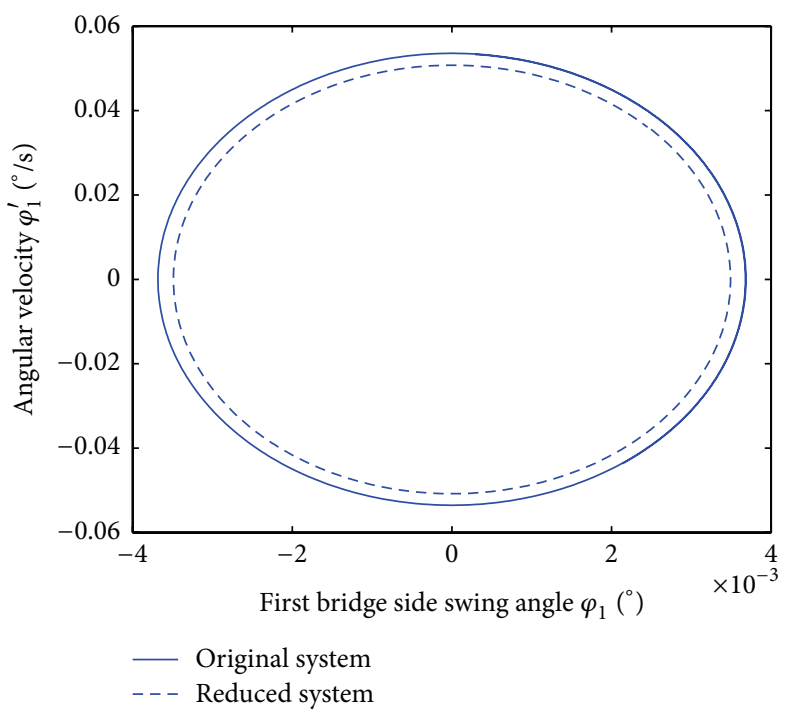

(e) The first bridge side pendulum

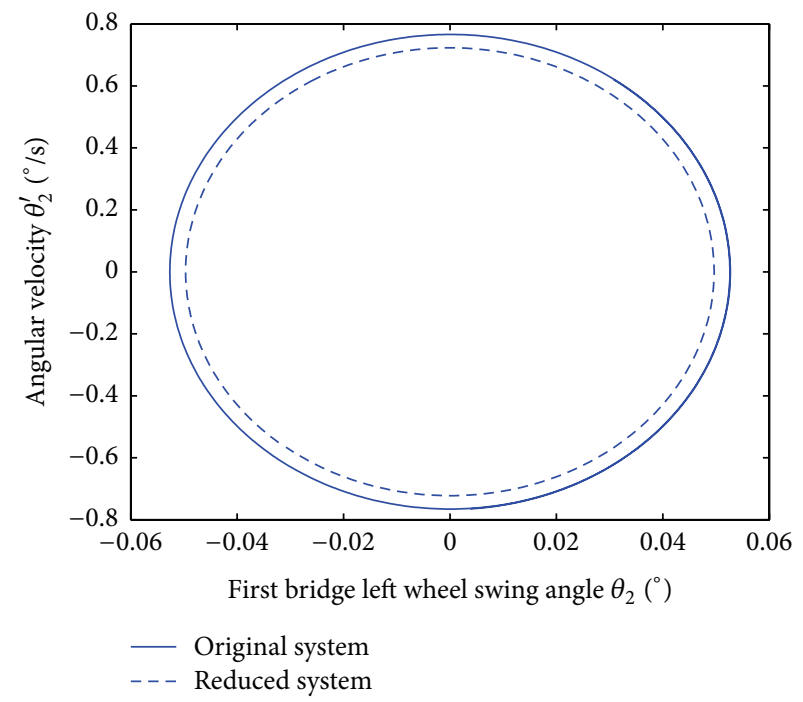

(b) First bridge left wheel

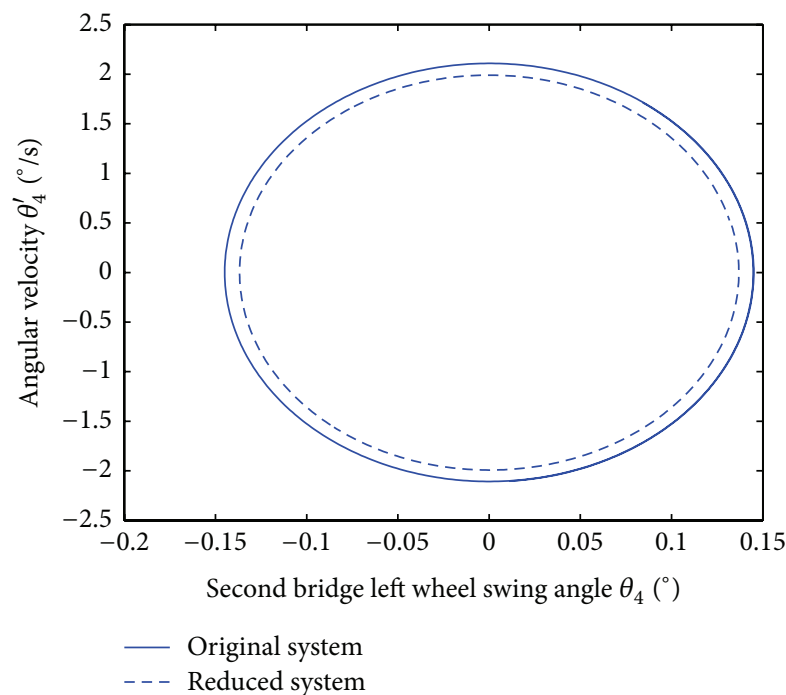

(d) Second bridge left wheel

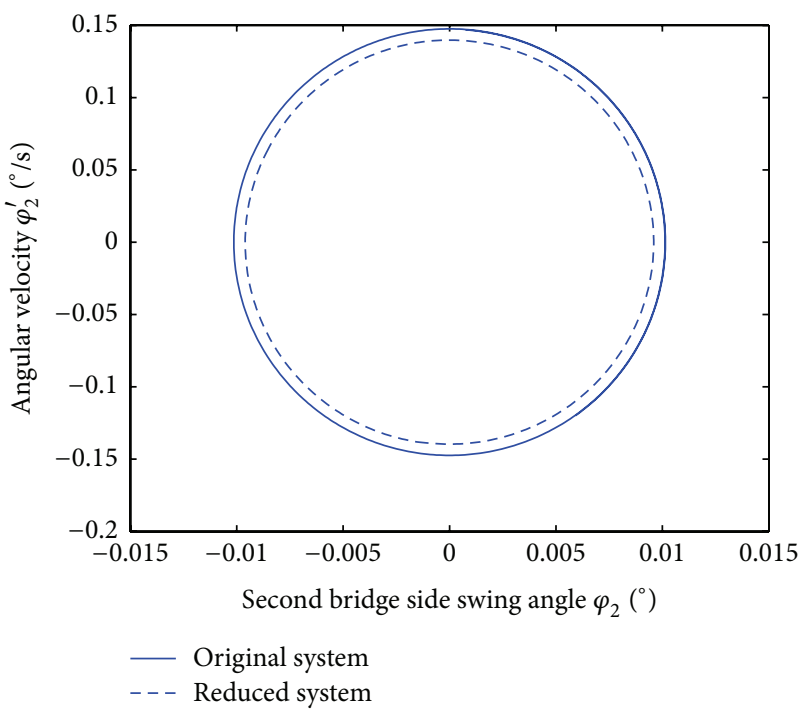

(f) The second bridge side pendulum

FIGURE 11: Continued. 


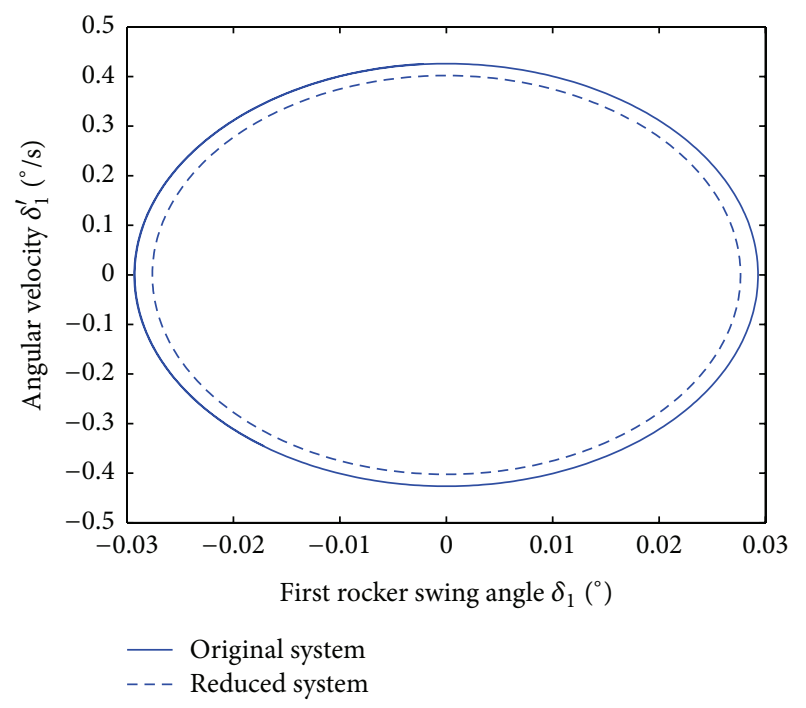

(g) First rocker swing angle

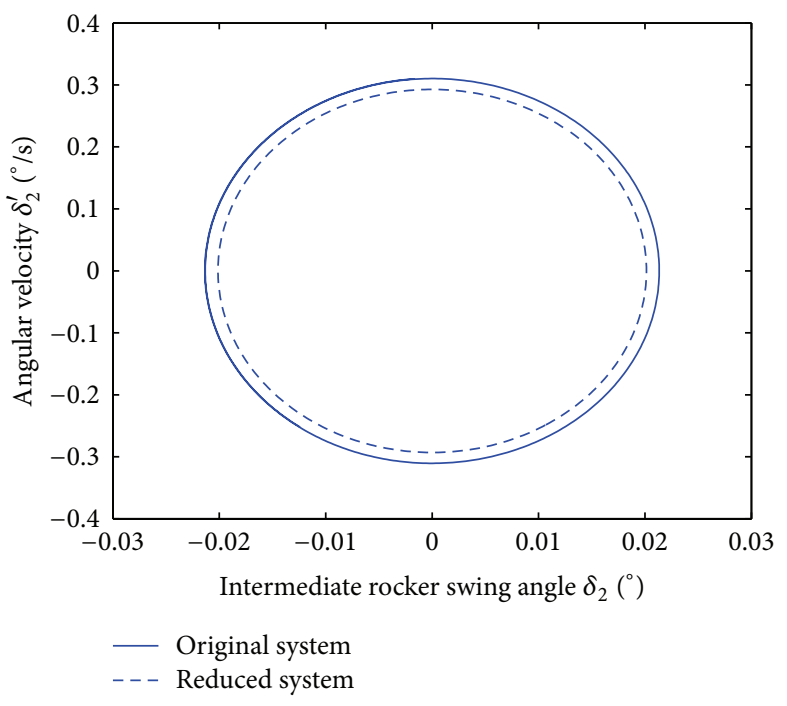

(h) Intermediate rocker swing angle

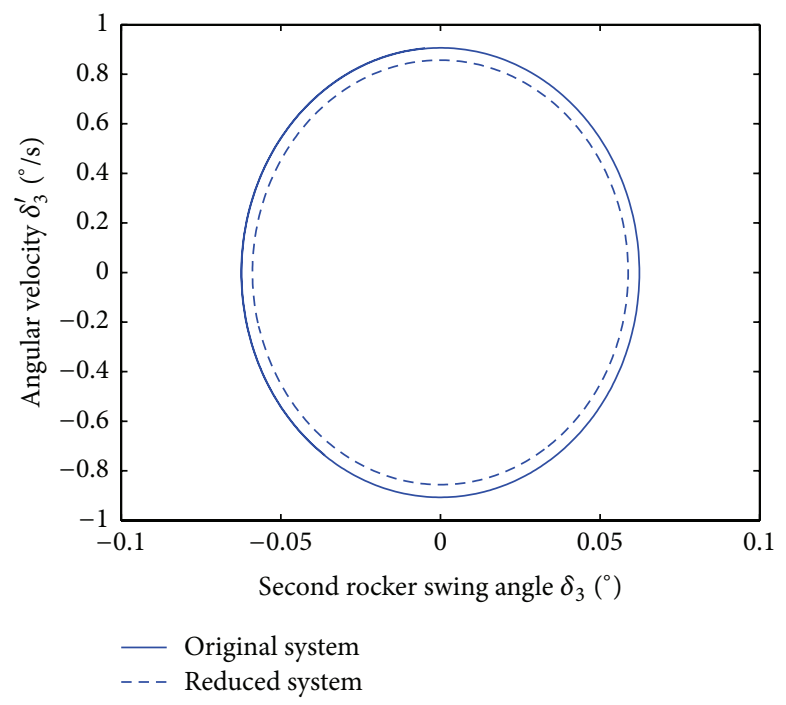

(i) Second rocker swing angle

FIGURE 11: For $v=40.8 \mathrm{~km} / \mathrm{h}$ comparison of limit cycle of original systems and limit cycle of dimensionality reduction system.

As shown in Figures 10 and 11, the dimension reduction system retains the bifurcation characteristics of the original system at the bifurcation point. Thus, we can utilize the limit cycle phase diagram of the bifurcation characteristics of the dimensionality reduction system near the bifurcation point.

4.3. Effect of Dry Friction Torque on Hopf Bifurcation Characteristics. We formulate the shimmy system differential equations considering the impact of kingpin clearance dry friction. Using the four- and five-order Runge-Kutta method, we generate the bifurcation diagrams of the shimmy characteristics of the first and second bridge wheels in the dualfront axle system, showing that the shimmy characteristics vary with velocity (see Figure 5 ). These diagrams are shown in Figure 12.

Figures 12(a) to 12(d) show that, with an increase in dry friction torque, the shimmy system speed bifurcation range, bifurcation curve amplitude, and the peak corresponding to the limit cycle all decrease.

Although increasing the dry friction can reduce the shimmy speed range and the system shimmy amplitude, it also reduces the steering performance and increases the wear between parts. Therefore, in designing suspension and steering systems, both steering agility and self-excited shimmy characteristics of the vehicle should be considered, aside from dry friction torque.

4.4. Effect of System Parameters on Shimmy. A heavy truck usually works under the conditions of overload and poor roads. Under these conditions, the dual-front axle steering system is subjected to tens of thousands $N$ force, and its lever components are usually up to a few meters. If the stiffness of the lever is designed unreasonably, the lever of a dualfront axle steering system inevitably becomes significantly 


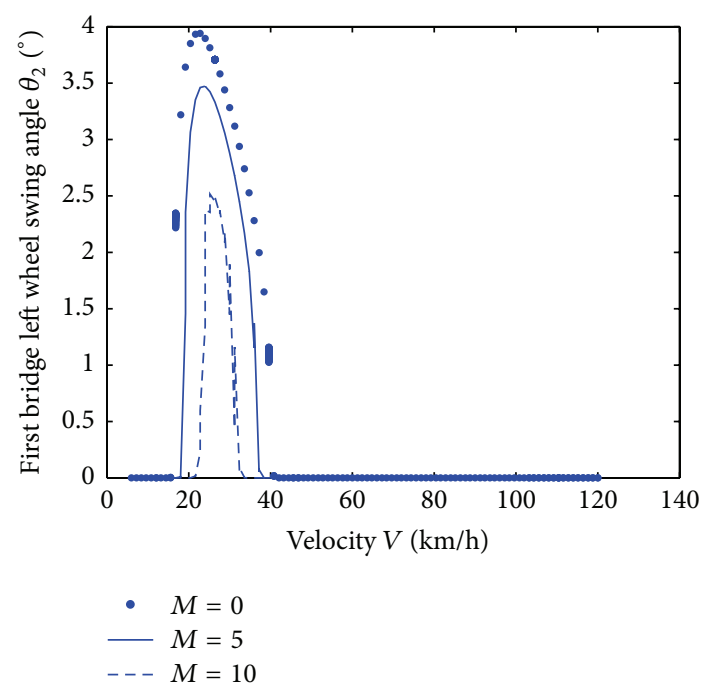

(a) First bridge left wheel

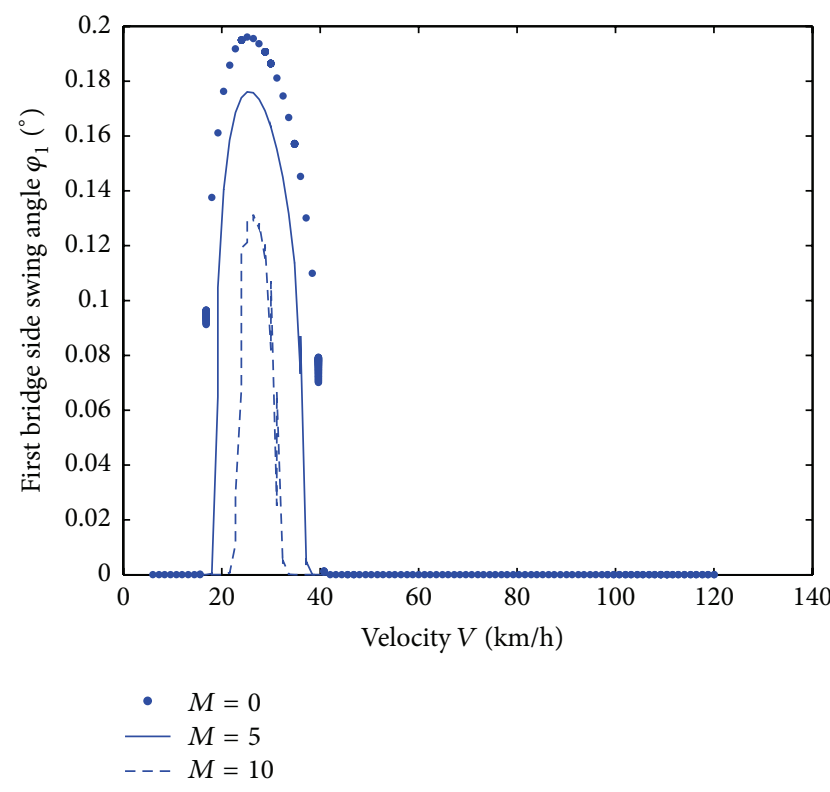

(c) The first bridge side pendulum

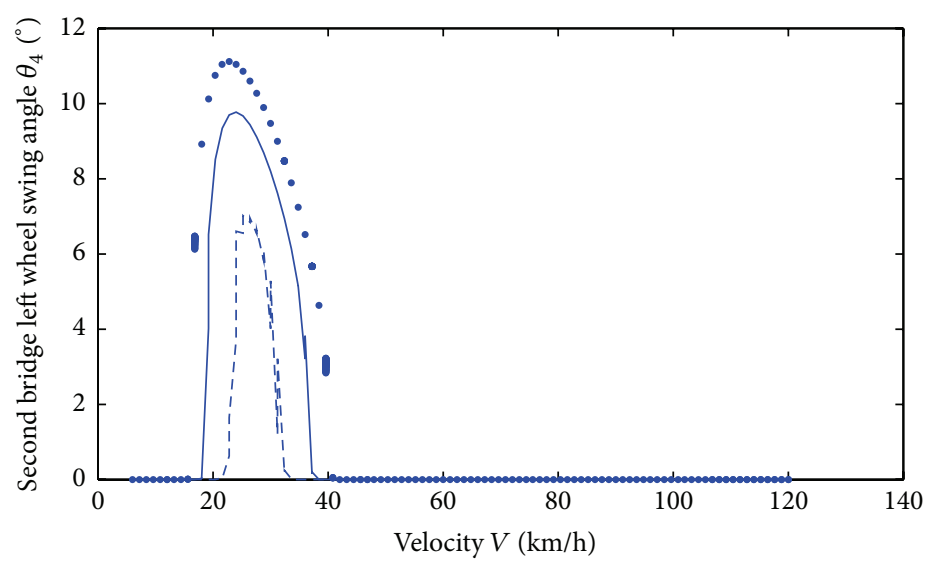

- $M=0$

$-M=5$

$--M=10$

(b) Second bridge left wheel

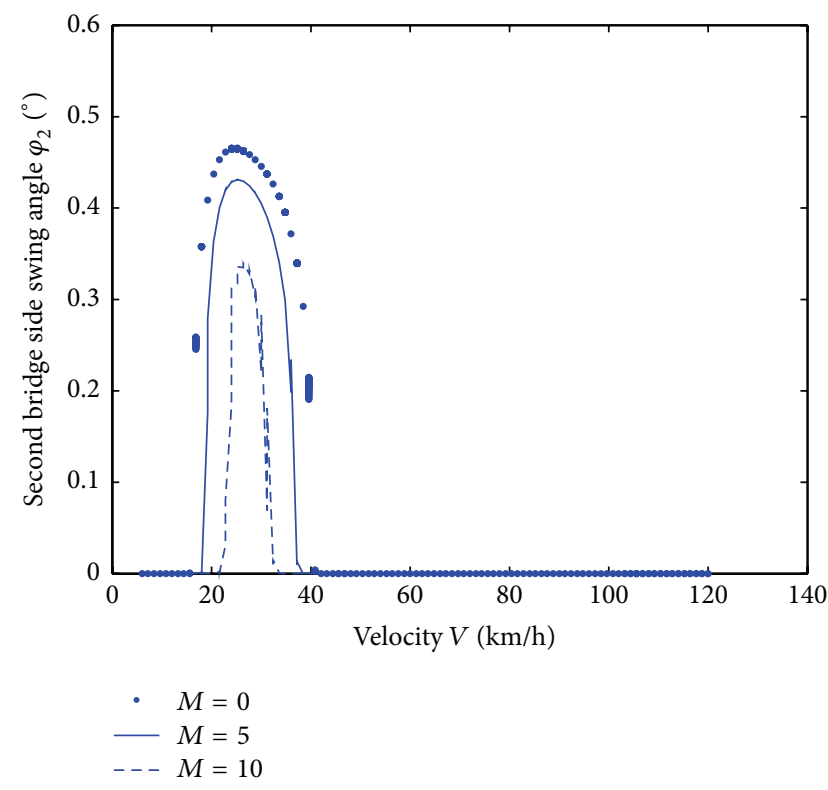

(d) The second bridge side pendulum

Figure 12: Influence of dry friction on the first and second bridge Hopf bifurcation characteristics.

deformed in the process of turning, and the deformation results in the shimmy and wear of tires. This phenomenon should not be ignored. The pneumatic trail $e$ and kingpin caster angle $\gamma$ are also important factors that affect shimmy. Figure 13 illustrates the influence factor of the first transition rod rigidity $k_{t 1}$, second transition rod rigidity $k_{t 2}$, pneumatic trail $e$, and kingpin caster angle $\gamma$ of the first and second bridge wheel shimmy characteristics, when $v=30 \mathrm{~km} / \mathrm{h}$.

Figure 13(a) shows that when the first transition rod stiffness increases from $20,000 \mathrm{~N} / \mathrm{m}$ to $100,000 \mathrm{~N} / \mathrm{m}$, the first bridge left wheel swing angle increases from $2.35^{\circ}$ to $3.47^{\circ}$, $\Delta \theta_{2}=1.12^{\circ}$, the second bridge left wheel swing angle decreases from $11.22^{\circ}$ to $8.89^{\circ}, \Delta \theta_{4}=-2.33^{\circ}$, the first bridge side pendulum angle increases from $0.15^{\circ}$ to $0.19^{\circ}, \Delta \theta_{2}=0.04^{\circ}$, the second bridge side pendulum angle decreases from $0.54^{\circ}$ to $0.42^{\circ}$, and $\Delta \theta_{4}=-0.12$. Figure $13(\mathrm{~b})$ shows that when the second transition rod stiffness increases from $20,000 \mathrm{~N} / \mathrm{m}$ to $100,000 \mathrm{~N} / \mathrm{m}$, the first bridge left wheel swing angle increases from $3.61^{\circ}$ to $1.58^{\circ}, \Delta \theta_{2}=2.03$, the second bridge left wheel swing angle decreases from $12.36^{\circ}$ to $6.72^{\circ}, \Delta \theta_{4}=-5.64^{\circ}$, the first bridge side pendulum angle increases from $0.11^{\circ}$ to $0.18^{\circ}$, $\Delta \theta_{2}=0.07^{\circ}$, the second bridge side pendulum angle decreases from $0.63^{\circ}$ to $0.32^{\circ}$, and $\Delta \theta_{4}=-0.31^{\circ}$. Thus, with an increase in transition rod stiffness $k_{t 1}$ and transition rod stiffness $k_{t 2}$, the first bridge left wheel swing angle and side pendulum angle increase, the second bridge left wheel swing angle and side pendulum angle decrease, and the second bridge left wheel swing angle and side pendulum angle significantly differ from those of the first bridge left wheel in amplitude. In addition, the effects of the second transition rod stiffness on the left 


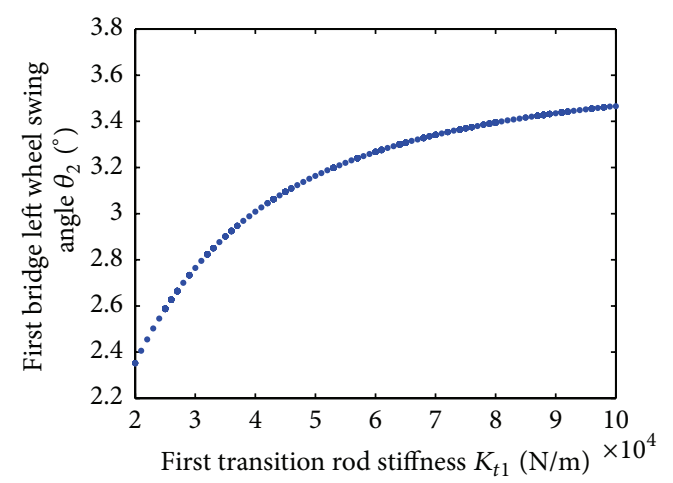

(1) First bridge left wheel

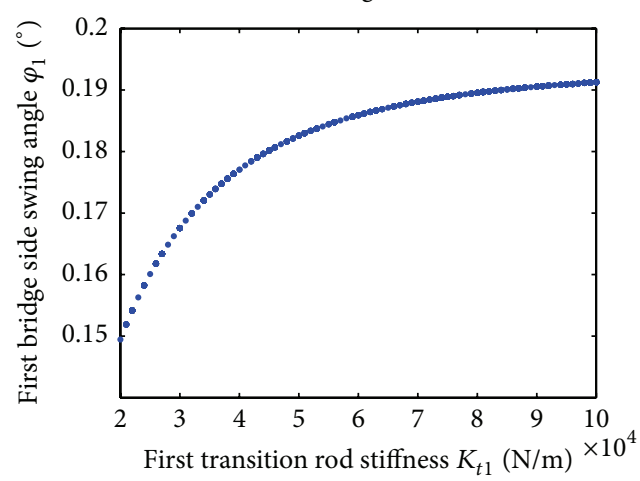

(3) The first bridge side pendulum

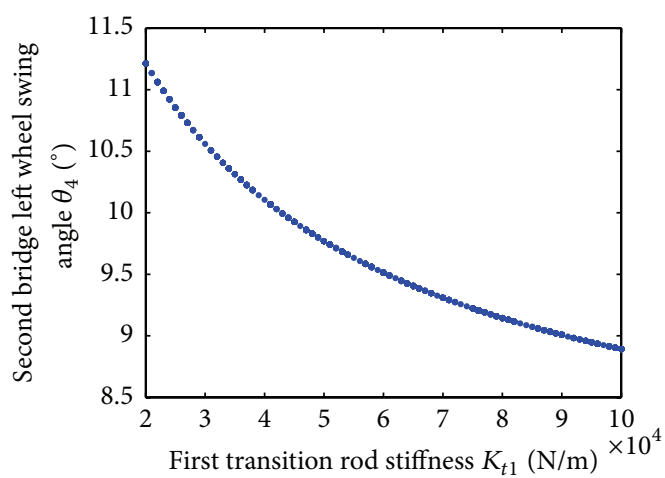

(2) Second bridge left wheel

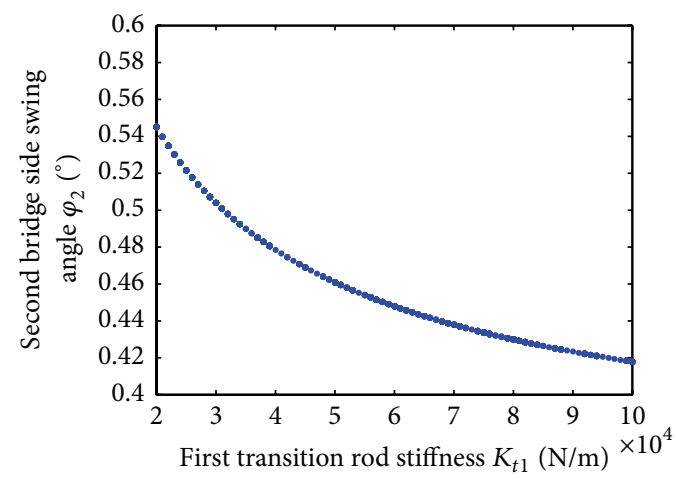

(4) The second bridge side pendulum

(a) Effect of the first transition rod stiffness $k_{t 1}$ on shimmy characteristics

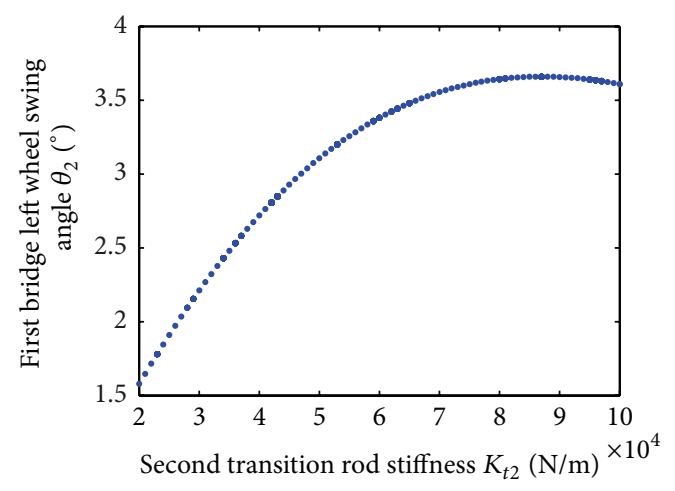

(1) First bridge left wheel

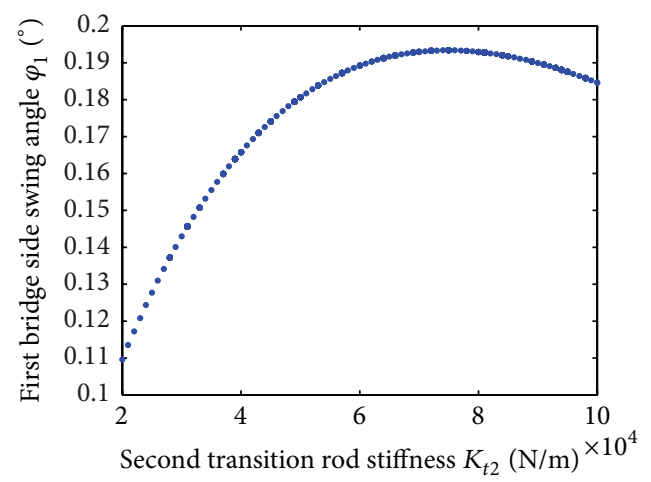

(3) The first bridge side pendulum

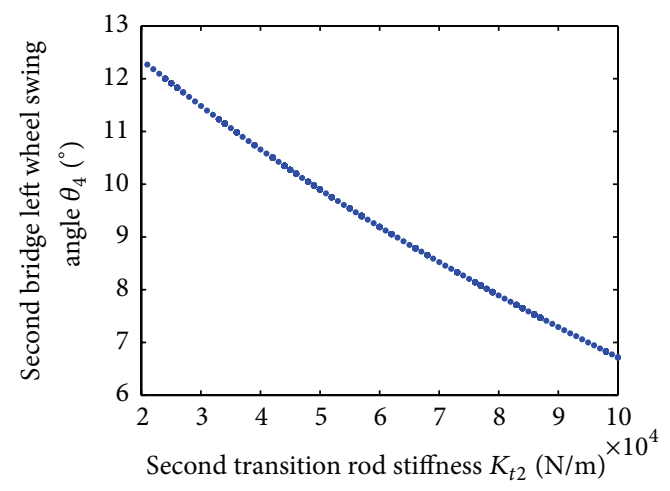

(2) Second bridge left wheel

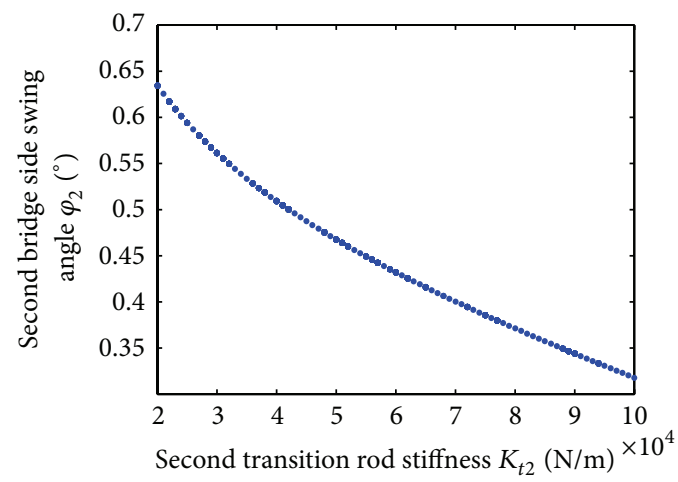

(4) The second bridge side pendulum

(b) Effect of the second transition rod stiffness $k_{t 2}$ on shimmy characteristics

Figure 13: Continued. 


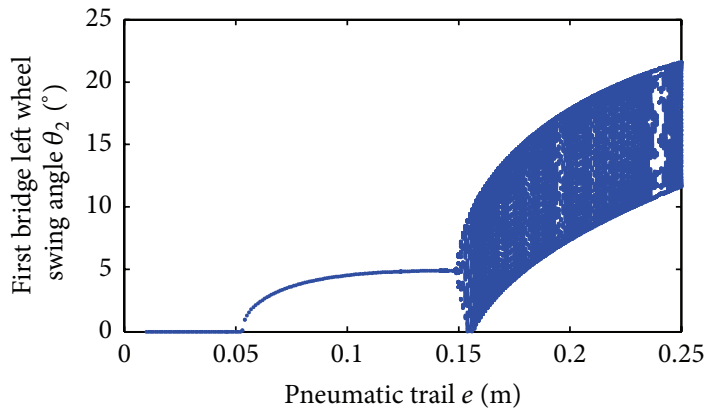

(1) First bridge left wheel

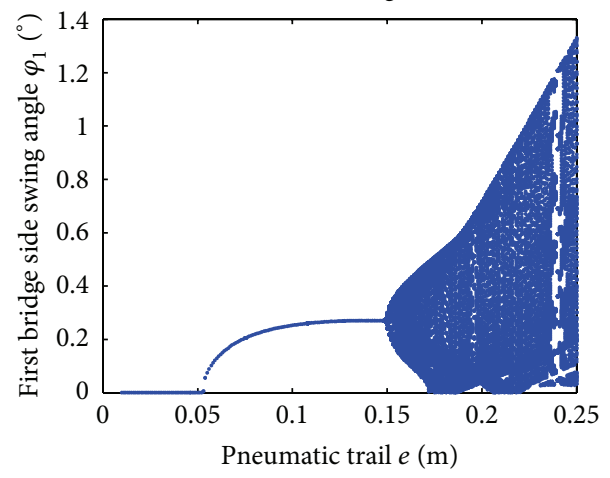

(3) The first bridge side pendulum

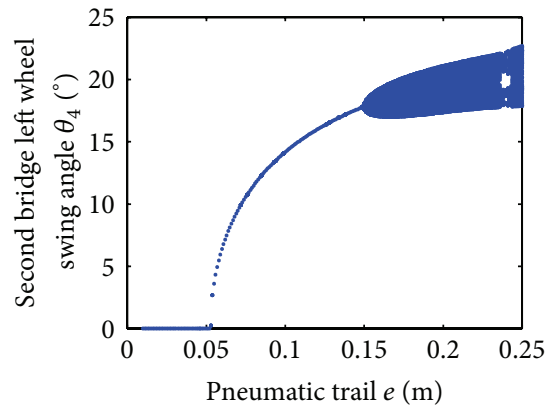

(2) Second bridge left wheel

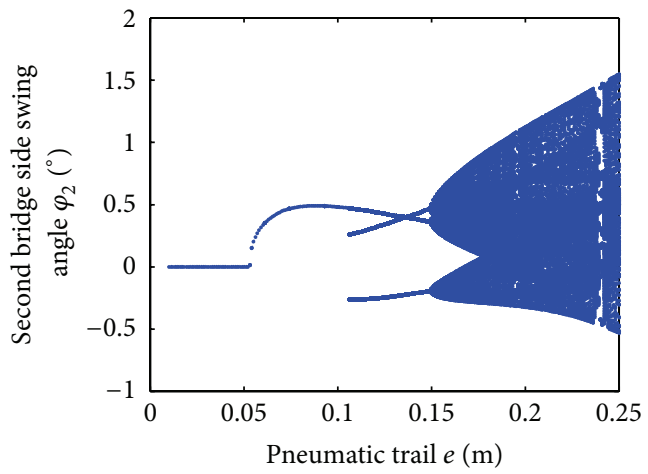

(4) The second bridge side pendulum

(c) Effect of the pneumatic trail $e$ on shimmy characteristics

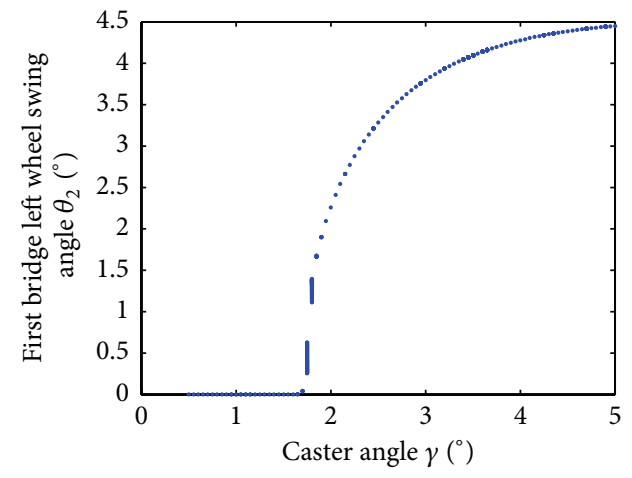

(1) First bridge left wheel

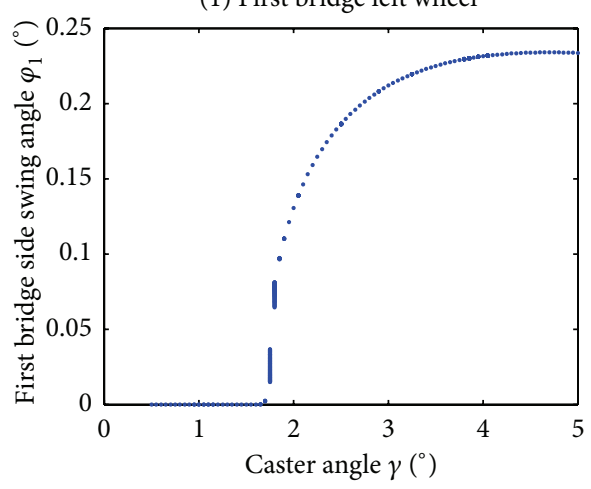

(3) The first bridge side pendulum

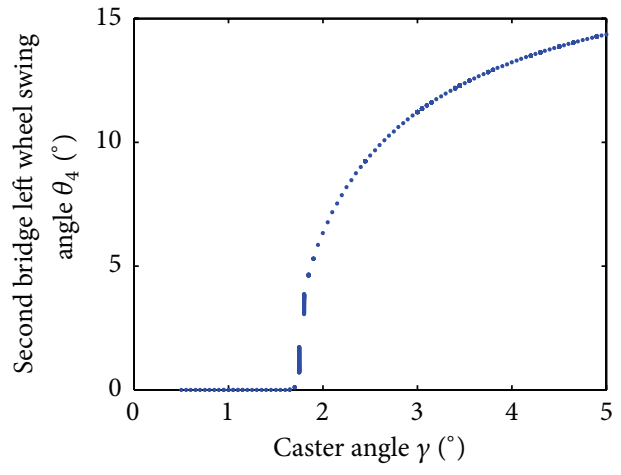

(2) Second bridge left wheel

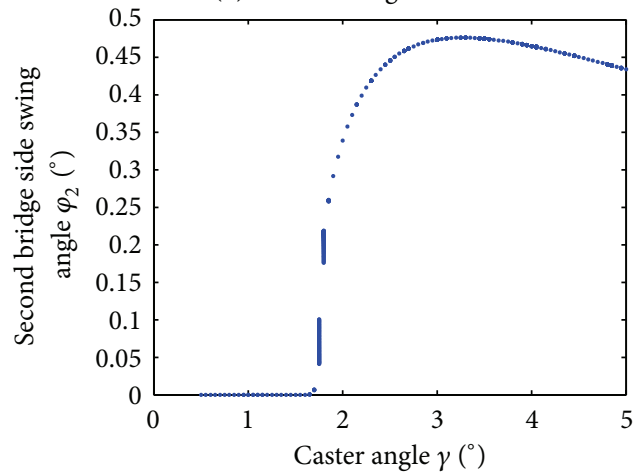

(4) The second bridge side pendulum

(d) Effect of caster angle on shimmy characteristics

FIGURE 13: Influence of system parameters. 
wheel swing angle and side pendulum angle magnitude of both bridges are significantly greater than those of the first transition rod stiffness.

Figure 13(c) shows that when the pneumatic trail $e$ increases from the minimum to 0.15 , the dual-front axle shimmy changes from steady into single-cycle limit cycle oscillation and ultimately to chaos, and the vibration amplitude becomes increasingly larger. Figure 13(d) shows that when the caster angle, $\gamma$, is less than 1.7, by the initial excitation, the system eventually stabilizes; however, when $\gamma$ increases to more than 1.7, the system state changes to the limit cycle oscillation. As $\gamma$ increases, the limit cycle increases.

Thus, to ensure that the tire wear in the two bridges is small and uniform, the stiffness of the first transition and second transition rods should be enhanced. However, considering the production process and production costs, designing a system with large transition rod stiffness is impossible. We can reduce or even eliminate, however, system shimmy by choosing a smaller pneumatic trail and kingpin caster angle.

\section{Conclusions}

(1) Based on the current widely used dual-front axle steering system in heavy trucks, we established a mechanics model for its dual bridge shimmy system mechanics and equations for its differential motion.

(2) Using Hopf bifurcation theorem and center manifold theory, we were able to determine the existence and stability of the shimmy system limit cycle. Using numerical method analysis, stable limit cycle characteristics at the critical speed point and bifurcation range were also determined. In conclusion, the sample vehicle dual-front axle shimmy is a self-excited vibration generated by Hopf bifurcation.

(3) In the dual-front axle shimmy system of heavy trucks, the shimmy intensities of the wheels of the first and second bridges are in a state of serious imbalance: the shimmy intensity at the second bridge is significantly greater than that at the first bridge. These findings coincide with the fact that the tire wear of the second bridge is always more severe than that of the first bridge in practice. Therefore, the mechanics model for the dual-axle shimmy system has high credibility and can simulate the sample vehicle during actual driving.

(4) The results of the methods of qualitative theory and those of the numerical methods have good consistency. The bifurcation characteristics of the shimmy system can be predicted using the methods of qualitative theory and can provide a theoretical reference for improving the design of a double-front axle vehicle.

(5) Speed is a bifurcation parameter of the vibration system, and dual-front axle steering transition rod stiffness is a sensitive parameter that affects system shimmy. Improving transition rod stiffness and selecting a smaller pneumatic trail and kingpin caster angle can reduce self-excited shimmy, reduce tire wear, and improve the driving stability and ride comfort of a dual-front axle vehicle.

\section{Conflict of Interests}

The authors declare that there is no conflict of interests regarding the publication of this paper.

\section{Acknowledgment}

This project is supported by the National Natural Science Foundation of China (Grant no. 51375130).

\section{References}

[1] K. Watanabe, J. Yamakawa, M. Tanaka, and T. Sasaki, "Turning characteristics of multi-axle vehicles," Journal of Terramechanics, vol. 44, no. 1, pp. 81-87, 2007.

[2] C. Mu, J. Yu, Y. Yang, and K. Wu, "Design for dual-front axle steering angle of the heavy truck," in Proceedings of the International Conference on Educational and Network Technology (ICENT '10), pp. 185-187, June 2010.

[3] J. Stuart, S. Cassara, B. Chan, and N. Augustyniak, "Recent experimental and simulation efforts to mitigate wobble and shimmy in commercial line haul vehicles," SAE International Journal of Commercial Vehicles, vol. 7, no. 2, pp. 366-380, 2014.

[4] H. B. Pacejka, Analysis of the Shimmy Phenomenon, Technische Hogeschool Delft, 1966.

[5] G. Dihua, H. Zeming, S. Jian et al., "Study on the vibration of vehicle's steering wheels," Automotive Engineering, vol. 2, pp. 29-40, 1984 (Chinese).

[6] S. Li and Y. Lin, "Study on the bifurcation character of steering wheel self-excited shimmy of motor vehicle," Vehicle System Dynamics, vol. 44, supplement 1, pp. 115-128, 2006.

[7] G. Jiang, Study of the Effect of Dry Friction on Multiple Limit Cycles in Shimmy, Hefei University of Technology, Hefei, China, 2012 (Chinese).

[8] Y. Gu, Z. Fang, G. Zhang, and Y. Qi, "Design of Heavy-duty truck multi-axle steering system," Automobile Technology, vol. 1, pp. 1-5, 2009 (Chinese).

[9] Y. Hou, Y. Hu, D. Hu, C. Li, and Y. Hou, "Synthesis of multi-axle steering system of heavy duty vehicle based on probability of steering angle," SAE Technical Paper 2000-01-3434, 2000.

[10] L. Wang, X. Liang, and P. Ji, "Reliability-based robust optimization on double-front-axle steering mechanism of trucks with clearances," Automobile Engineering, vol. 1, pp. 90-93, 2014 (Chinese).

[11] Z. Xu, Y. He, H. Yin et al., "Study of steering wheel shimmy of LT1080 truck crane," Engineering Machinery, vol. 3, pp. 7-11, 1994 (Chinese).

[12] R. L. Nisonger and D. N. Wormley, "Dynamic performance of automated guideway transit vehicles with dual-axle steering," IEEE Transactions on Vehicular Technology, vol. 28, no. 1, pp. 88-94, 1979.

[13] D. H. Wu and J. H. Lin, "Analysis of dynamic lateral response for a multi-axle-steering tractor and trailer," Heavy Vehicle Systems, vol. 10, no. 4, pp. 281-294, 2003.

[14] D. E. Williams, "Generalised multi-axle vehicle handling," Vehicle System Dynamics, vol. 50, no. 1, pp. 149-166, 2012. 
[15] M. Demić, "Analysis of influence of design parameters on steered wheels shimmy of heavy vehicles," Vehicle System Dynamics, vol. 26, no. 5, pp. 343-379, 1996.

[16] D. J. Cole and D. Cebon, "Truck suspension design to minimize road damage," Proceedings of the Institution of Mechanical Engineers. Part D, vol. 210, no. 2, pp. 95-107, 1996.

[17] Y. Chen, "Double axle steering auto tire abnormal wear of 'internal research' and 'external research"' Equipment Manufacturing Technology, vol. 11, pp. 113-116, 2011 (Chinese).

[18] D. Liao, Optimized Design and Simulation Study for DualFront Axle Steering System of Heavy Trucks, Hunan University, Changsha, China, 2012 (Chinese).

[19] L. Li, C. Zhang, Z. Wang, M. Liu, and J. Shi, "Motion simulation and optimization design of double-front axle steering system based on ADAMS," in Proceedings of the IEEE Transportation Electrification Conference and Expo (ITEC '14), pp. 1-5, IEEE, Beijing, China, September 2014.

[20] H. Li, Steering and Handling Stability Simulation of MultiAxle, Wuhan University of Technology, Wuhan, China, 2013, (Chinese).

[21] J. Q. Shen and Z. J. Jing, "A new detecting method for conditions of existence of Hopf bifurcation," Acta Mathematicae Applicatae Sinica, vol. 11, no. 1, pp. 79-93, 1995.

[22] H. Pacejka, "A new tyre model with an application in vehicle dynamics studies," SAE Paper 89007, SAE International, 1989.

[23] H. Hu and Z. Wu, "Stability and Hopf bifurcation of four-wheelsteering vehicles involving driver's delay," Nonlinear Dynamics, vol. 22, no. 4, pp. 361-374, 2000.

[24] K. Guo, D. Lu, S.-K. Chen, W. C. Lin, and X.-P. Lu, "The UniTire model: a nonlinear and non-steady-state tyre model for vehicle dynamics simulation," Vehicle System Dynamics, vol. 43, supplement 1, pp. 341-358, 2005.

[25] G. Gim and P. E. Nikravesh, "An analytical model of pneumatic tyres for vehicle dynamic simulations. Part 1: pure slips," International Journal of Vehicle Design, vol. 11, no. 6, pp. 589618, 1990.

[26] D. Wei, P. Wang, Z. Pan, S. Hu, and H. Xiao, "Study of the effects upon vehicle stability exerted by tie rod end clearance under slalom maneuver," SAE International Journal of Passenger Car, vol. 7, no. 1, pp. 395-404, 2014.

[27] P. Tao, A Study on Control of Nonlinear Stability for TractorSemitrailer, Jilin University, Jilin, China, 2012 (Chinese).

[28] R. S. Sharp, "A comparison of tyre representation in a simple wheel shimmy problem," Vehicle System Dynamics, vol. 9, no. 1, pp. $45-47,1980$.

[29] A. Y. T. Leung, Z. Qichang, and C. Yushu, "Normal form analysis of hopf bifurcation exemplified by Duffing's equation," Shock and Vibration, vol. 1, no. 3, pp. 233-240, 1994. 

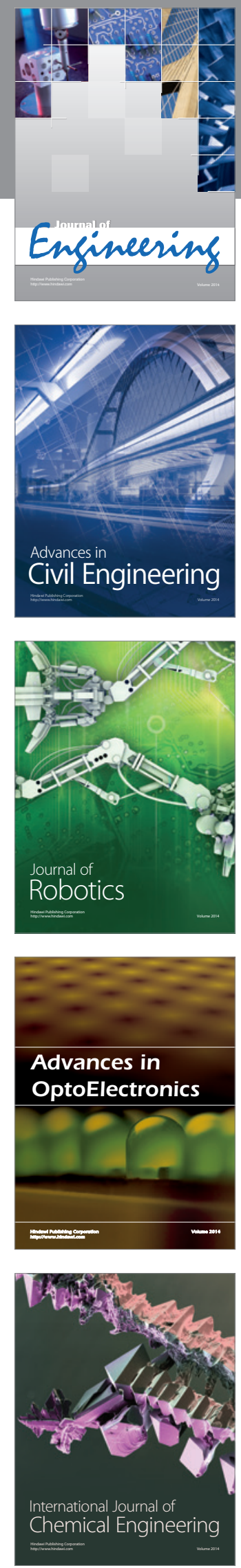

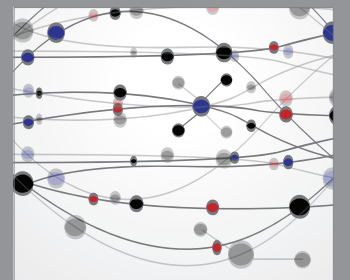

The Scientific World Journal
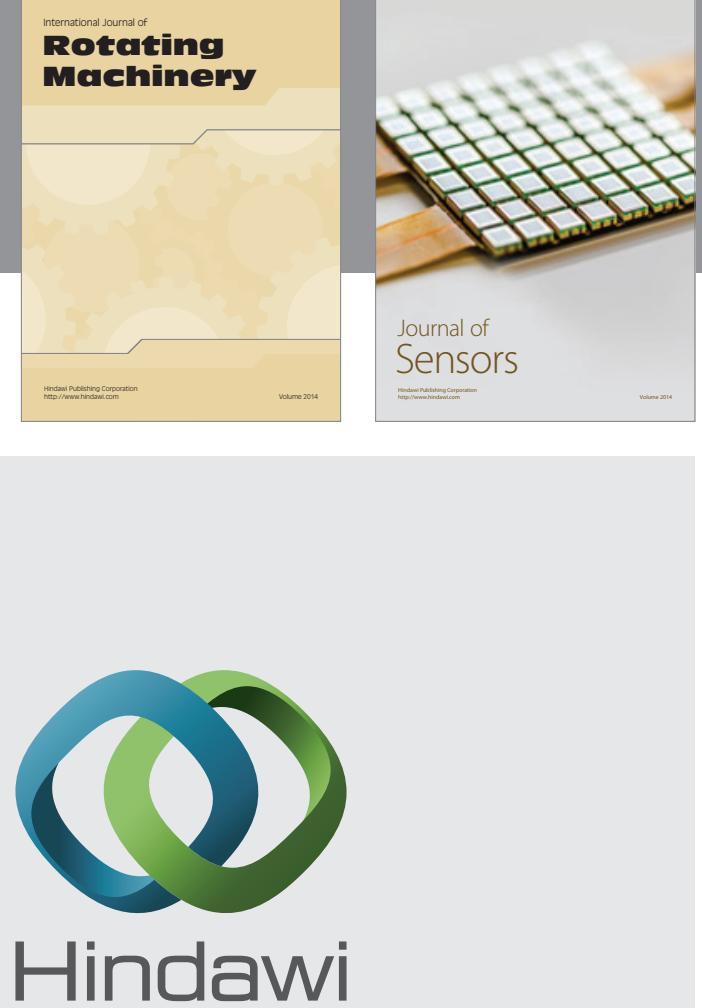

Submit your manuscripts at http://www.hindawi.com
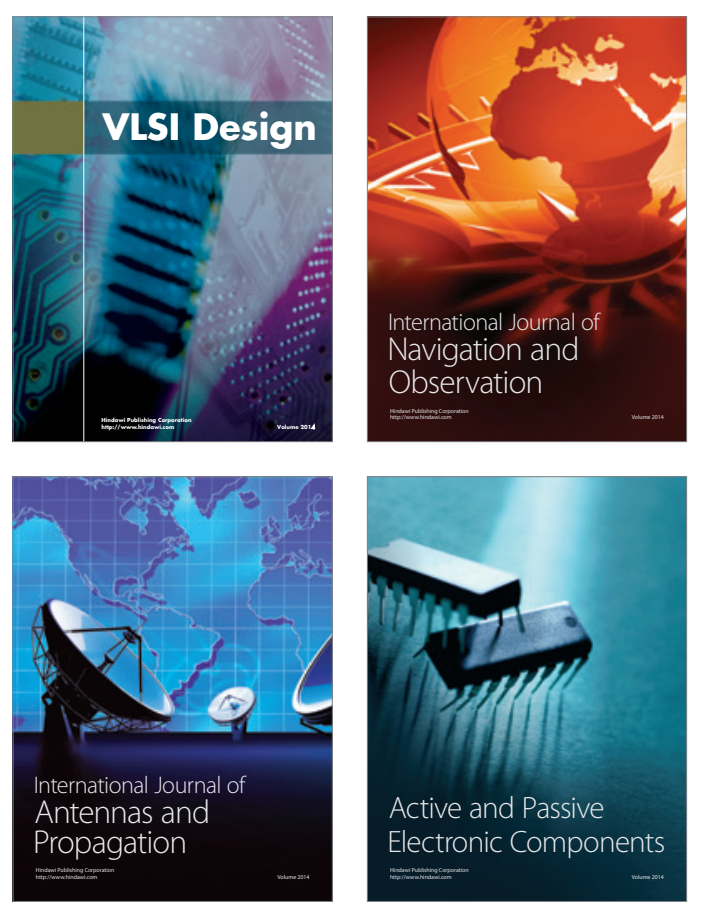
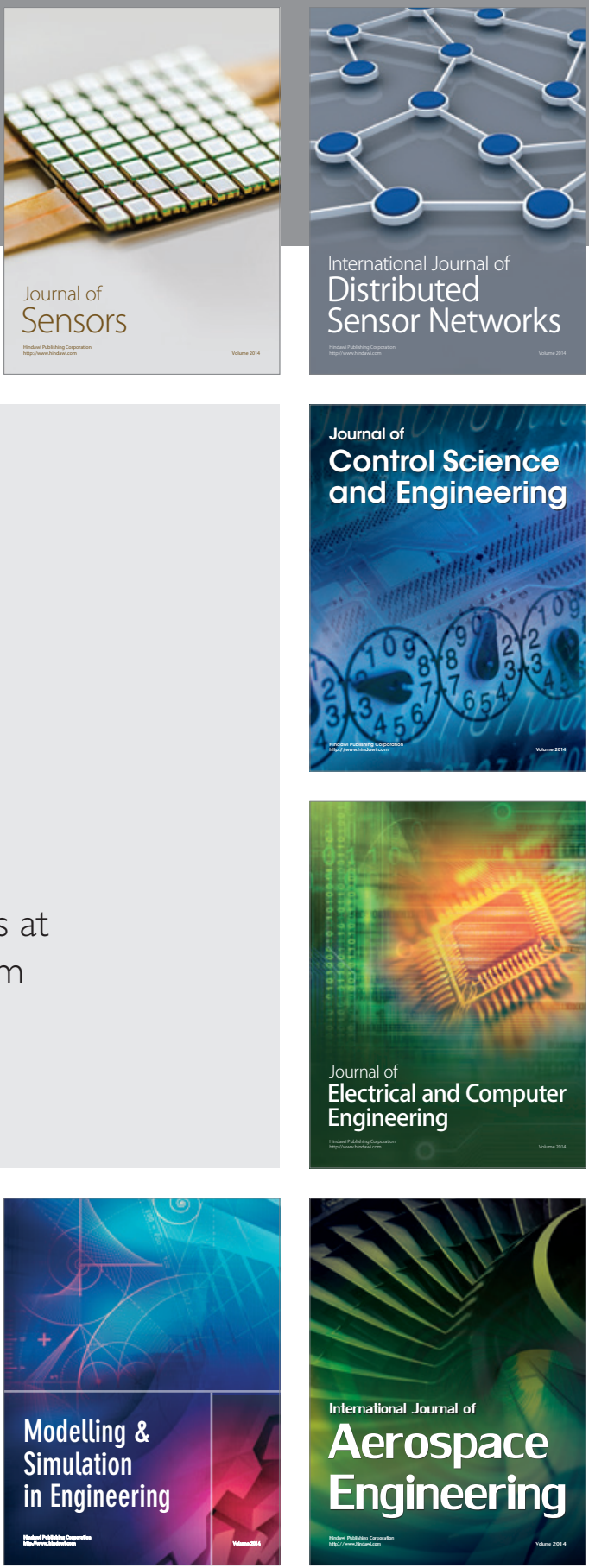

Journal of

Control Science

and Engineering
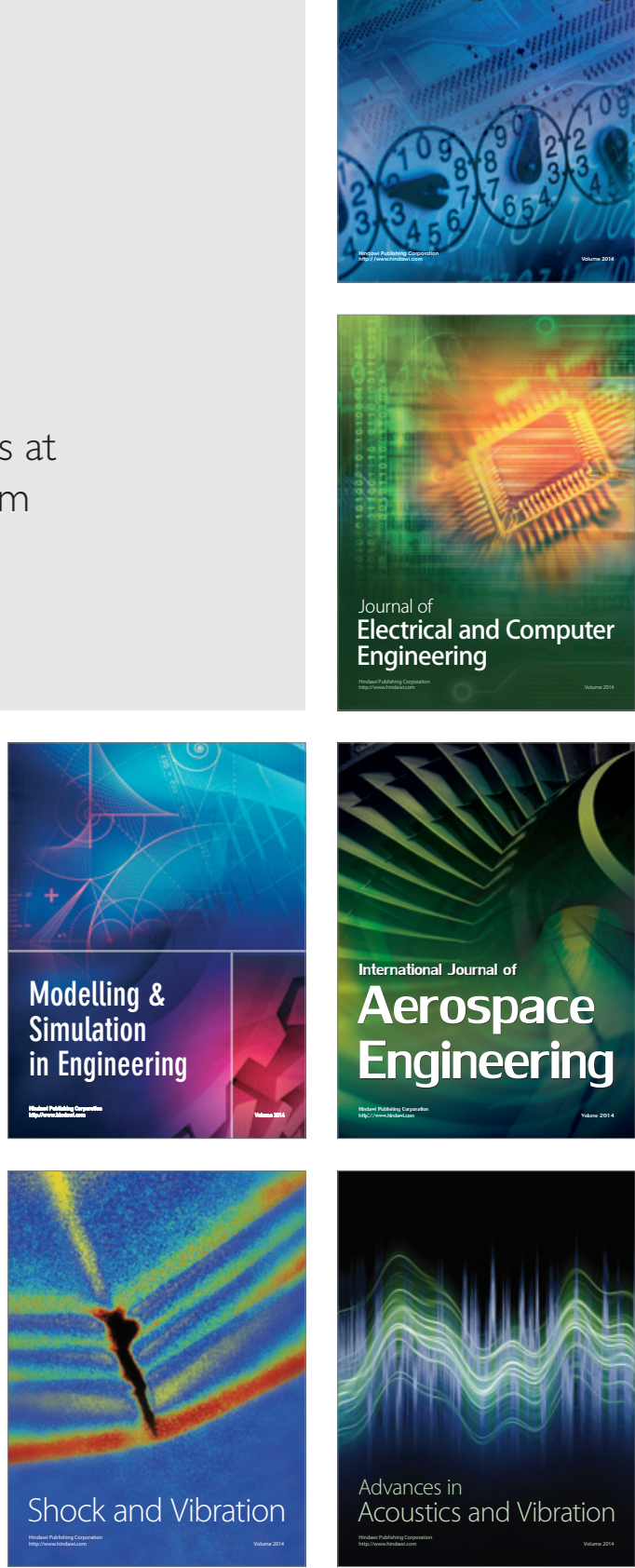